EUROPEAN ORGANIZATION FOR NUCLEAR RESEARCH

CERN-PPE/97-013

January 28, 1997

\title{
Measurement of the Spectral Functions of Vector Current Hadronic Tau Decays
}

\author{
The ALEPH Collaboration ${ }^{1}$
}

\begin{abstract}
A measurement of the spectral functions of non-strange $\tau$ vector current final states is presented, using $124358 \tau$ pairs recorded by the ALEPH detector at LEP during the years 1991 to 1994 . The spectral functions of the dominant two- and four-pion $\tau$ decay channels are compared to published results of $e^{+} e^{-}$annihilation experiments via isospin rotation. A combined fit of the pion form factor from $\tau$ decays and $e^{+} e^{-}$data is performed using different parametrizations. The mass and the width of the $\rho^{ \pm}(770)$ and the $\rho^{0}(770)$ are separately determined in order to extract possible isospin violating effects. The mass and width differences are measured to be $M_{\rho^{ \pm}(770)}-M_{\rho^{0}(770)}=(0.0 \pm 1.0) \mathrm{MeV} / c^{2}$ and $\Gamma_{\rho^{ \pm}(770)}-\Gamma_{\rho^{0}(770)}=$ $(0.1 \pm 1.9) \mathrm{MeV} / c^{2}$.
\end{abstract}

(To be submitted to Zeitschrift für Physik)

${ }^{1}$ See next pages for the list of authors. 


\section{The ALEPH Collaboration}

R. Barate, D. Buskulic, D. Decamp, P. Ghez, C. Goy, J.-P. Lees, A. Lucotte, M.-N. Minard, J.-Y. Nief, B. Pietrzyk

Laboratoire de Physique des Particules (LAPP), IN² $P^{3}$-CNRS, 74019 Annecy-le-Vieux Cedex, France

M.P. Casado, M. Chmeissani, P. Comas, J.M. Crespo, M. Delfino, E. Fernandez, M. Fernandez-Bosman, Ll. Garrido, ${ }^{15}$ A. Juste, M. Martinez, R. Miquel, Ll.M. Mir, S. Orteu, C. Padilla, I.C. Park, A. Pascual, J.A. Perlas, I. Riu, F. Sanchez, F. Teubert

Institut de Fisica d'Altes Energies, Universitat Autonoma de Barcelona, 08193 Bellaterra (Barcelona), Spain $^{7}$

A. Colaleo, D. Creanza, M. de Palma, G. Gelao, G. Iaselli, G. Maggi, M. Maggi, N. Marinelli, S. Nuzzo, A. Ranieri, G. Raso, F. Ruggieri, G. Selvaggi, L. Silvestris, P. Tempesta, A. Tricomi, ${ }^{3}$ G. Zito

Dipartimento di Fisica, INFN Sezione di Bari, 70126 Bari, Italy

X. Huang, J. Lin, Q. Ouyang, T. Wang, Y. Xie, R. Xu, S. Xue, J. Zhang, L. Zhang, W. Zhao

Institute of High-Energy Physics, Academia Sinica, Beijing, The People's Republic of China ${ }^{8}$

D. Abbaneo, R. Alemany, U. Becker, A.O. Bazarko, ${ }^{20}$ P. Bright-Thomas, M. Cattaneo, F. Cerutti, H. Drevermann, R.W. Forty, M. Frank, R. Hagelberg, J. Harvey, P. Janot, B. Jost, E. Kneringer, J. Knobloch, I. Lehraus, G. Lutters, P. Mato, A. Minten, L. Moneta, A. Pacheco, J.-F. Pusztaszeri, F. Ranjard, P. Rensing, ${ }^{12}$ G. Rizzo, L. Rolandi, D. Schlatter, M. Schmitt, O. Schneider, W. Tejessy, I.R. Tomalin, H. Wachsmuth, A. Wagner

European Laboratory for Particle Physics (CERN), 1211 Geneva 23, Switzerland

Z. Ajaltouni, A. Barrès, C. Boyer, A. Falvard, C. Ferdi, P. Gay, C . Guicheney, P. Henrard, J. Jousset, B. Michel, S. Monteil, J-C. Montret, D. Pallin, P. Perret, F. Podlyski, J. Proriol, P. Rosnet, J.M. Rossignol

Laboratoire de Physique Corpusculaire, Université Blaise Pascal, $I N^{2} P^{3}$-CNRS, Clermont-Ferrand, 63177 Aubière, France

T. Fearnley, J.B. Hansen, J.D. Hansen, J.R. Hansen, P.H. Hansen, B.S. Nilsson, B. Rensch, A. Wäänänen Niels Bohr Institute, 2100 Copenhagen, Denmark ${ }^{9}$

G. Daskalakis, A. Kyriakis, C. Markou, E. Simopoulou, I. Siotis, A. Vayaki

Nuclear Research Center Demokritos (NRCD), Athens, Greece

A. Blondel, G. Bonneaud, J.C. Brient, P. Bourdon, A. Rougé, M. Rumpf, A. Valassi, ${ }^{6}$ M. Verderi, H. Videau

Laboratoire de Physique Nucléaire et des Hautes Energies, Ecole Polytechnique, IN $N^{2} P^{3}-C N R S, 91128$

Palaiseau Cedex, France

D.J. Candlin, M.I. Parsons

Department of Physics, University of Edinburgh, Edinburgh EH9 3JZ, United Kingdom ${ }^{10}$

E. Focardi, G. Parrini, K. Zachariadou

Dipartimento di Fisica, Università di Firenze, INFN Sezione di Firenze, 50125 Firenze, Italy

M. Corden, C. Georgiopoulos, D.E. Jaffe

Supercomputer Computations Research Institute, Florida State University, Tallahassee, FL 323064052, USA ${ }^{13,14}$ 
A. Antonelli, G. Bencivenni, G. Bologna, ${ }^{4}$ F. Bossi, P. Campana, G. Capon, D. Casper, V. Chiarella, G. Felici, P. Laurelli, G. Mannocchi, ${ }^{5}$ F. Murtas, G.P. Murtas, L. Passalacqua, M. Pepe-Altarelli

Laboratori Nazionali dell'INFN (LNF-INFN), 00044 Frascati, Italy

L. Curtis, S.J. Dorris, A.W. Halley, I.G. Knowles, J.G. Lynch, V. O'Shea, C. Raine, J.M. Scarr, K. Smith, P. Teixeira-Dias, A.S. Thompson, E. Thomson, F. Thomson, R.M. Turnbull

Department of Physics and Astronomy, University of Glasgow, Glasgow G12 8QQ,United Kingdom ${ }^{10}$

C. Geweniger, G. Graefe, P. Hanke, G. Hansper, V. Hepp, E.E. Kluge, A. Putzer, M. Schmidt, J. Sommer, K. Tittel, S. Werner, M. Wunsch

Institut für Hochenergiephysik, Universität Heidelberg, 69120 Heidelberg, Fed. Rep. of Germany ${ }^{16}$

R. Beuselinck, D.M. Binnie, W. Cameron, P.J. Dornan, M. Girone, S. Goodsir, E.B. Martin, A. Moutoussi, J. Nash, J.K. Sedgbeer, A.M. Stacey, M.D. Williams

Department of Physics, Imperial College, London SW7 2BZ, United Kingdom ${ }^{10}$

G. Dissertori, P. Girtler, D. Kuhn, G. Rudolph

Institut für Experimentalphysik, Universität Innsbruck, 6020 Innsbruck, Austria ${ }^{18}$

A.P. Betteridge, C.K. Bowdery, P. Colrain, G. Crawford, A.J. Finch, F. Foster, G. Hughes, T. Sloan, M.I. Williams

Department of Physics, University of Lancaster, Lancaster LA1 4YB, United Kingdom ${ }^{10}$

A. Galla, I. Giehl, A.M. Greene, C. Hoffmann, K. Jakobs, K. Kleinknecht, G. Quast, B. Renk, E. Rohne, H.-G. Sander, P. van Gemmeren, C. Zeitnitz

Institut für Physik, Universität Mainz, 55099 Mainz, Fed. Rep. of Germany ${ }^{16}$

J.J. Aubert, C. Benchouk, A. Bonissent, G. Bujosa, D. Calvet, J. Carr, P. Coyle, C. Diaconu, F. Etienne, N. Konstantinidis, O. Leroy, F. Motsch, P. Payre, D. Rousseau, M. Talby, A. Sadouki, M. Thulasidas, K. Trabelsi

Centre de Physique des Particules, Faculté des Sciences de Luminy, IN ${ }^{2} P^{3}$-CNRS, 13288 Marseille, France

M. Aleppo, F. Ragusa ${ }^{2}$

Dipartimento di Fisica, Università di Milano e INFN Sezione di Milano, 20133 Milano, Italy

R. Berlich, W. Blum, V. Büscher, H. Dietl, F. Dydak, ${ }^{2}$ G. Ganis, C. Gotzhein, H. Kroha, G. Lütjens, G. Lutz, W. Männer, H.-G. Moser, R. Richter, A. Rosado-Schlosser, S. Schael, R. Settles, H. Seywerd, R. St. Denis, H. Stenzel, W. Wiedenmann, G. Wolf

Max-Planck-Institut für Physik, Werner-Heisenberg-Institut, 80805 München, Fed. Rep. of Germany ${ }^{16}$

J. Boucrot, O. Callot, ${ }^{2}$ S. Chen, Y. Choi,${ }^{21}$ A. Cordier, M. Davier, L. Duflot, J.-F. Grivaz, Ph. Heusse, A. Höcker, A. Jacholkowska, M. Jacquet, D.W. Kim ${ }^{24}$ F. Le Diberder, J. Lefrançois, A.-M. Lutz, I. Nikolic, M.-H. Schune, S. Simion, E. Tournefier, J.-J. Veillet, I. Videau, D. Zerwas

Laboratoire de l'Accélérateur Linéaire, Université de Paris-Sud, IN ${ }^{2} P^{3}-C N R S, 91405$ Orsay Cedex, France

P. Azzurri, G. Bagliesi, G. Batignani, S. Bettarini, C. Bozzi, G. Calderini, M. Carpinelli, M.A. Ciocci, V. Ciulli, R. Dell'Orso, R. Fantechi, I. Ferrante, L. Foà, ${ }^{1}$ F. Forti, A. Giassi, M.A. Giorgi, A. Gregorio, F. Ligabue, A. Lusiani, P.S. Marrocchesi, A. Messineo, F. Palla, G. Sanguinetti, A. Sciabà, P. Spagnolo, J. Steinberger, R. Tenchini, G. Tonelli, ${ }^{19}$ C. Vannini, A. Venturi, P.G. Verdini

Dipartimento di Fisica dell'Università, INFN Sezione di Pisa, e Scuola Normale Superiore, 56010 Pisa, Italy

G.A. Blair, L.M. Bryant, J.T. Chambers, Y. Gao, M.G. Green, T. Medcalf, P. Perrodo, J.A. Strong, J.H. von Wimmersperg-Toeller

Department of Physics, Royal Holloway \& Bedford New College, University of London, Surrey TW20 OEX, United Kingdom ${ }^{10}$ 
D.R. Botterill, R.W. Clifft, T.R. Edgecock, S. Haywood, P. Maley, P.R. Norton, J.C. Thompson, A.E. Wright

Particle Physics Dept., Rutherford Appleton Laboratory, Chilton, Didcot, Oxon OX11 OQX, United Kingdom ${ }^{10}$

B. Bloch-Devaux, P. Colas, S. Emery, W. Kozanecki, E. Lançon, M.C. Lemaire, E. Locci, P. Perez, J. Rander, J.-F. Renardy, A. Roussarie, J.-P. Schuller, J. Schwindling, A. Trabelsi, B. Vallage

CEA, DAPNIA/Service de Physique des Particules, CE-Saclay, 91191 Gif-sur-Yvette Cedex, France ${ }^{17}$

S.N. Black, J.H. Dann, R.P. Johnson, H.Y. Kim, A.M. Litke, M.A. McNeil, G. Taylor

Institute for Particle Physics, University of California at Santa Cruz, Santa Cruz, CA 95064, USA ${ }^{22}$

C.N. Booth, R. Boswell, C.A.J. Brew, S. Cartwright, F. Combley, M.S. Kelly, M. Lehto, W.M. Newton, J. Reeve, L.F. Thompson

Department of Physics, University of Sheffield, Sheffield S3 7RH, United Kingdom ${ }^{10}$

A. Böhrer, S. Brandt, G. Cowan, C. Grupen, P. Saraiva, L. Smolik, F. Stephan

Fachbereich Physik, Universität Siegen, 57068 Siegen, Fed. Rep. of Germany ${ }^{16}$

M. Apollonio, L. Bosisio, R. Della Marina, G. Giannini, B. Gobbo, G. Musolino

Dipartimento di Fisica, Università di Trieste e INFN Sezione di Trieste, 34127 Trieste, Italy

J. Rothberg, S. Wasserbaech

Experimental Elementary Particle Physics, University of Washington, WA 98195 Seattle, U.S.A.

S.R. Armstrong, E. Charles, P. Elmer, D.P.S. Ferguson, Y.S. Gao, ${ }^{23}$ S. González, T.C. Greening, O.J. Hayes, H. Hu, S. Jin, P.A. McNamara III, J.M. Nachtman, J. Nielsen, W. Orejudos, Y.B. Pan, Y. Saadi, I.J. Scott, J. Walsh, Sau Lan Wu, X. Wu, J.M. Yamartino, G. Zobernig

Department of Physics, University of Wisconsin, Madison, WI 53706, USA ${ }^{11}$

\footnotetext{
${ }^{1}$ Now at CERN, 1211 Geneva 23, Switzerland.

${ }^{2}$ Also at CERN, 1211 Geneva 23, Switzerland.

${ }^{3}$ Also at Dipartimento di Fisica, INFN, Sezione di Catania, Catania, Italy.

${ }^{4}$ Also Istituto di Fisica Generale, Università di Torino, Torino, Italy.

${ }^{5}$ Also Istituto di Cosmo-Geofisica del C.N.R., Torino, Italy.

${ }^{6}$ Supported by the Commission of the European Communities, contract ERBCHBICT941234.

${ }^{7}$ Supported by CICYT, Spain.

${ }^{8}$ Supported by the National Science Foundation of China.

${ }^{9}$ Supported by the Danish Natural Science Research Council.

${ }^{10}$ Supported by the UK Particle Physics and Astronomy Research Council.

${ }^{11}$ Supported by the US Department of Energy, grant DE-FG0295-ER40896.

${ }^{12}$ Now at Dragon Systems, Newton, MA 02160, U.S.A.

${ }^{13}$ Supported by the US Department of Energy, contract DE-FG05-92ER40742.

${ }^{14}$ Supported by the US Department of Energy, contract DE-FC05-85ER250000.

${ }^{15}$ Permanent address: Universitat de Barcelona, 08208 Barcelona, Spain.

${ }^{16}$ Supported by the Bundesministerium für Bildung, Wissenschaft, Forschung und Technologie, Fed.

Rep. of Germany.

${ }^{17}$ Supported by the Direction des Sciences de la Matière, C.E.A.

${ }^{18}$ Supported by Fonds zur Förderung der wissenschaftlichen Forschung, Austria.

${ }^{19}$ Also at Istituto di Matematica e Fisica, Università di Sassari, Sassari, Italy.

${ }^{20}$ Now at Princeton University, Princeton, NJ 08544, U.S.A.

${ }^{21}$ Permanent address: Sung Kyun Kwan University, Suwon, Korea.

${ }^{22}$ Supported by the US Department of Energy, grant DE-FG03-92ER40689.

${ }^{23}$ Now at Harvard University, Cambridge, MA 02138, U.S.A.

${ }^{24}$ Permanent address: Kangnung National University, Kangnung, Korea.
} 


\section{Introduction}

The spectral functions of vector current hadronic $\tau$ decays are related to the isovector cross section of electron-positron annihilation [1] if isospin invariance of the hadronic currents (Conserved Vector Current property - CVC) is valid. In this respect, data on hadronic $\tau$ decays provide not only an additional and precise measurement, but also an independent test of theoretical and experimental consistencies. This is even more important as $e^{+} e^{-}$data for those final states with higher pion multiplicity are in some cases rather inconsistent. Finally, and most importantly, $\tau$ spectral functions are an important probe for the study of perturbative and non-perturbative QCD.

A total number of $124358 \mathrm{Z}$ boson decays into $\tau$ pairs were accumulated by the ALEPH detector at LEP during the years 1991 to 1994. These events, in addition to the high reconstruction efficiency of exclusive $\tau$ decay modes, allow a precise measurement of the spectral functions of the dominant vector hadronic $\tau$ decays including those with high neutral pion multiplicity.

The measurement of the non-strange $\tau$ vector spectral functions is presented, with special emphasis on the dominant two- and four-pion final states. In addition, extensive comparisons to isovector $e^{+} e^{-}$annihilation results are performed. The pion form factor in $\tau^{-} \rightarrow \pi^{-} \pi^{0} \nu_{\tau}^{2}$ decays is fitted using parametrizations of Kühn-Santamaria [2] and Gounaris-Sakurai [3]. Additionally, $e^{+} e^{-}$and $\tau$ data are combined in fitting the masses and widths of the $\rho^{ \pm}(770)$ and $\rho^{0}(770)$ separately in order to extract information about isospin violation between these states.

Several analyses have already been performed along these lines. In 1987, the ARGUS Collaboration published the spectral function of the decay $\tau^{-} \rightarrow 2 \pi^{-} \pi^{+} \pi^{0} \nu_{\tau}$ [4]. The CLEO Collaboration compared $e^{+} e^{-}$results via CVC to the corresponding invariant mass spectra of the $\tau$ final states $2 \mathrm{~h}^{-} \mathrm{h}^{+} \pi^{0}$ and $3 \mathrm{~h}^{-} 2 \mathrm{~h}^{+} \pi^{0}$ [5]. Recently, the ALEPH Collaboration published a study of $\tau$ decays into $\eta$ and $\omega$ mesons [6], in which the final states $\pi^{-} \pi^{0} \eta$ and $\pi^{-} \omega$ were found to be in good agreement with $e^{+} e^{-}$data.

The paper is organized as follows: after the definition of the $\tau$ vector spectral functions and its relationship to $e^{+} e^{-}$annihilation data, a brief introduction of the ALEPH experiment is given and the $\tau$ selection and particle identification procedure are described as well as the $\tau$ decay classification including the photon and neutral pion reconstruction. Then the measurement of the spectral functions, which are directly related to the invariant mass spectra of the respective $\tau$ decay channels, is presented. This is an important step in the analysis since the unfolding of detector effects requires particular care. A detailed description of systematic effects affecting the measurements follows. The results are compared to the published data of $e^{+} e^{-}$annihilation experiments and, in particular, a fit of the pion form factor is performed.

More tests concerning QCD phenomenology, in particular precise fits of the strong coupling constant $\alpha_{s}$ and non-perturbative contributions, tests of QCD sum rules and the measurement of the axial-vector spectral functions will follow in forthcoming papers.

\footnotetext{
${ }^{2}$ Throughout this paper, charge conjugate states are implied.
} 
Preliminary results have already been presented in Ref. [7].

\section{Spectral Functions}

The measurement of the non-strange $\tau$ vector current hadronic spectral functions requires the selection and identification of $\tau$ decay modes with a $\mathrm{G}$-parity $\mathrm{G}=+1$, i.e., hadronic channels with an even number of pions, neutral or charged. The isovector spectral function $v_{1, X^{-}}(s)$ of a $\tau$ vector channel $X^{-} \nu_{\tau}$ is obtained by dividing the normalized invariant masssquared distribution $\left(1 / N_{X^{-}}\right)\left(d N_{X^{-}} / d s\right)$ for a given hadronic mass $\sqrt{s}$ by the appropriate kinematic factor:

$$
v_{1, X^{-}}(s)=H \frac{B\left(\tau^{-} \rightarrow X^{-} \nu_{\tau}\right)}{B\left(\tau^{-} \rightarrow e^{-} \nu_{\tau} \bar{\nu}_{e}\right)} \frac{1}{N_{X^{-}}} \frac{d N_{X^{-}}}{d s}\left[\left(1-\frac{s}{M_{\tau}^{2}}\right)^{2}\left(1+\frac{2 s}{M_{\tau}^{2}}\right)\right]^{-1}
$$

with the branching ratio $B\left(\tau^{-} \rightarrow X^{-} \nu_{\tau}\right)$ normalized to the branching ratio of the electron channel. The dimensional constant $H$ is defined as

$$
H=\frac{M_{\tau}^{2}}{6\left|V_{u d}\right|^{2} S_{\mathrm{EW}}},
$$

where $\left|V_{u d}\right|=0.9752 \pm 0.0007$ denotes the CKM weak mixing matrix element [8] and $S_{\mathrm{EW}}=1.0194$ accounts for electroweak radiative corrections [9]. The $\tau$ mass $M_{\tau}=1776.96_{-0.27}^{+0.31} \mathrm{MeV} / c^{2}$ is taken from the recent BES measurement [10].

Theoretically, the spectral functions of hadronic $\tau$ decays are related via dispersion relations to the imaginary parts of the analytic two-point correlation functions $\Pi_{i j, U}^{\mu \nu}(q) \equiv$ $i \int d^{4} x e^{i q x}\left\langle 0\left|T\left(U_{i j}^{\mu}(x) U_{i j}^{\nu}(0)^{\dagger}\right)\right| 0\right\rangle$ of vector $\left(U \equiv V=\bar{\psi}_{j} \gamma^{\mu} \psi_{i}\right)$ or axial-vector $(U \equiv$ $A=\bar{\psi}_{j} \gamma^{\mu} \gamma_{5} \psi_{i}$ ) colour-singlet quark currents in corresponding quantum states (see, e.g., Ref. $[11,12])$.

Then, using the optical theorem and isospin rotation, the spectral function of a $\tau$ decay mode $X^{-} \nu_{\tau}$ in a given isospin state for the hadronic system is related to the $e^{+} e^{-}$ annihilation cross section of the corresponding isovector final state $X^{0}$ :

$$
\sigma_{e^{+} e^{-} \rightarrow X^{0}}^{I=1}=\frac{4 \pi \alpha^{2}}{s} v_{1, X^{-}} .
$$

For the two-body hadronic final state $X^{-} \equiv \pi^{-} \pi^{0}$ in $\tau$ decays, it is customary to introduce a weak pion form factor $\tilde{F}_{\pi}^{I=1}$ to describe phenomenologically the probability density of the transition $\mathrm{W}^{-} \rightarrow \pi^{-} \pi^{0}$ :

$$
\left|\tilde{F}_{\pi}^{I=1}(s)\right|^{2}=\frac{12}{\beta_{\pi}^{3}(s)} v_{1, \pi^{-} \pi^{0}}(s),
$$

where $\beta_{\pi}(s)=\left(1-4 m_{\pi}^{2} / s\right)^{1 / 2}$ is the pion velocity in the hadronic centre of mass. The weak pion form factor can be identified with the isovector electromagnetic form factor, given by

$$
\left|F_{\pi}^{I=1}(s)\right|^{2}=\frac{3}{\pi} \frac{s}{\alpha^{2} \beta_{\pi}^{3}(s)} \sigma_{e^{+} e^{-} \rightarrow \pi^{+} \pi^{-}}^{I=1},
$$

using isospin invariance (CVC). 


\section{The ALEPH Detector}

The ALEPH detector provides both tracking and calorimetric information over almost the full solid angle. The features relevant for this analysis are briefly mentioned here, while a detailed description of its components and performance can be found in Refs. [13, 14].

The momentum of charged particles is reconstructed using the information given by the three tracking devices immersed in a $1.5 \mathrm{~T}$ axial magnetic field: a double-sided silicon microstrip vertex detector, an eight-layer axial wire chamber and a large time projection chamber (TPC), the last providing up to 21 space points for tracks of charged particles and up to 338 measurements of the ionization loss $(d E / d x)$. The transverse momentum resolution achieved in the combined fit is $\Delta p_{\mathrm{T}} / p_{\mathrm{T}} \simeq\left(5 \oplus 0.6 p_{\mathrm{T}}(\mathrm{GeV} / c)^{-1}\right) \times 10^{-3}$.

The electromagnetic calorimeter (ECAL), located inside the magnetic coil, is formed of a barrel surrounding the TPC, closed at each end by an endcap. It consists of 45 layers of a total thickness of 22 radiation lengths. The energy and position of a shower is read out using cathode pads with dimensions $3 \times 3 \mathrm{~cm}^{2}$, arranged to form towers pointing to the interaction zone; each tower is read out in three segments in depth corresponding respectively to 4, 9, and 9 radiation lengths. The energy resolution is $\Delta E / E \simeq 18 \% / \sqrt{E /(\mathrm{GeV})}+0.9 \%$. There are 74000 such towers, corresponding to an average granularity of $0.9^{\circ} \times 0.9^{\circ}$. The inactive zones ("cracks") between the ECAL modules represent $2 \%$ of the total solid angle in the barrel and $6 \%$ in the endcaps. The fine granularity and the longitudinal segmentation of the calorimeter play an important role in the photon and neutral pion reconstruction, and in the identification of fake photons produced by hadronic interactions of charged hadrons or split-offs from electromagnetic showers.

The hadron calorimeter (HCAL) has 23 layers of iron absorber each $5 \mathrm{~cm}$ thick with limited streamer tubes $9 \times 9 \mathrm{~mm}^{2}$ in cross section between each layer. The tower readout is built from pads with an angular size of $3.7^{\circ} \times 3.7^{\circ}$. Strips running along the tubes provide a digital readout giving a two-dimensional view of the development of hadronic showers and muon trajectories.

The trigger efficiency is measured to be better than $99.99 \%$ within the selection cuts of this analysis.

Tau pair events produced at the $\mathrm{Z}$ mass peak are simulated using the standard Monte Carlo program KORALZ $[15,16,17]$ and passed through a full detector simulation based on GEANT [18]. Electromagnetic showers are simulated according to parametrizations obtained from test beam data [13]. Several tests and corrections of the detector simulation have been carried out within the scope of this analysis to assure its reliability and to estimate systematic uncertainties. 


\section{Event Selection and Classification}

\section{1 $\tau$ Pair Selection}

The topology of $\tau$ pair events produced at the $\mathrm{Z}$ mass scale is characterized by back-toback, narrow jets with an average multiplicity much lower than for hadronic $\mathrm{Z}$ decays. Thus, candidates are selected by retaining low multiplicity events coming mainly from lepton pair decays of the Z. A detailed description of the $\tau$ pair pre-selection can be found in Ref. [19]. Additional cuts are applied in order to suppress Bhabha and dimuon background as well as background coming from two-photon processes and cosmic ray events [20]. More details about the cuts to remove hadronic $\mathrm{Z}$ decays from the $\tau$ pair sample are provided in Ref. [21]. The analysis presented here is based on a sample of data recorded by the ALEPH detector during the years 1991 up to 1994 of LEP running. In total $124358 \tau$ pairs are selected, with a detection efficiency of $(78.8 \pm 0.1) \%$. The overall non- $\tau$ background contribution in the hadronic modes, obtained from Monte Carlo simulation corrected with data $[20,21]$, amounts to $(0.6 \pm 0.2) \%$.

Tau decays are classified according to the number of charged hadrons and to the number of reconstructed $\pi^{0}$ 's.

\subsection{Charged Particle Identification}

To identify charged particles coming from $\tau$ decays, a maximum likelihood method is employed, combining different and essentially uncorrelated information measured for each individual track. This procedure is originally described in Ref. [22] and additionally improved in Ref. [20]. As a result, hadrons from one-prong $\tau$ decays with a momentum above $2 \mathrm{GeV} / c$ are correctly identified with an efficiency of $(98.31 \pm 0.06) \%$. The probabilities of electrons and muons being misidentified as hadrons are $(0.51 \pm 0.10) \%$ and $(0.68 \pm 0.10) \%$, respectively.

\subsection{Photons and Neutral Pion Reconstruction}

Photon identification and $\pi^{0}$ reconstruction, briefly described in this section, and the subsequent $\tau$ decay classification follow in detail the procedure of Ref. [21].

Photons have characteristic shower profiles in the electromagnetic calorimeter. They are reconstructed by collecting associated energetic ECAL towers, forming a cluster. To distinguish genuine photons from fake photons a likelihood method is applied using ECAL information, e.g., the fraction of energy in the respective ECAL stacks, the transverse size of the shower or the distance between the barycentre of the cluster and the closest charged track, to veto fake photon candidates. This procedure is performed for all photons in a given hemisphere, attributing a probability to be a genuine photon to each of them. A more detailed description of the likelihood and the variables used can be found in Ref. [21].

In order to identify in an ensemble of reconstructed photons those originating from a $\pi^{0}$ decay, probabilities are calculated as the product of the genuine photon probabilities and a probability that the pair originates from a $\pi^{0}$ using a $\pi^{0}$ mass constraint. Neutral pions 
reconstructed with two photons are called resolved. At higher $\pi^{0}$ energy, the opening angle between the boosted photons tends to become smaller than the calorimeter resolution so that the two electromagnetic showers are often merged in one cluster. The transverse energy distribution in the ECAL nevertheless allows the computation of energy-weighted moments providing a measure of the two-photon invariant mass. Such reconstructed $\pi^{0}$ 's are called unresolved. A remaining photon considered as originating from a $\pi^{0}$ while the second photon has been lost is called residual.

To improve the energy resolution of resolved $\pi^{0}$ s, the energies of the contributing photons and the opening angle are refitted using the nominal $\pi^{0}$ mass as a constraint. The reconstructed energies and momenta of identified $\pi^{0}$ 's are subjected to a certain number of tests and corrections to assure the reliability of the simulation. These are described in Ref. [21] and Section 6 as far as crucial points of this analysis are concerned.

\section{Measurement of the $\tau$ Vector Spectral Functions}

The measurement of the $\tau$ spectral functions defined in Eq. (1) requires the determination of the physical invariant mass-squared distribution. To extract it from the measured one it needs to be unfolded from the effects of measurement distortion. The unfolding procedure used in this analysis follows a new method published in Ref. [23], which is briefly recalled here.

\subsection{The Unfolding Procedure}

The convolution (folding) of an invariant mass distribution during the measurement process can be understood as a linear equation

$$
A x=b,
$$

where $x=x_{1}, \ldots, x_{n}$ is the unknown (binned) true mass distribution to be determined, $A=A_{11}, \ldots, A_{m n}$ is the detector response matrix taken from the simulation of the measurement process, and $b=b_{1}, \ldots, b_{m}$ is the measured distribution. The matrix element $A_{i j}$ gives the probability that an event with a true mass in bin $j$ is reconstructed in bin $i$. Since the detector response matrix is a numerically singular matrix, the direct inversion of (6) leads to very unstable and therefore useless results. To extract the statistically significant information in A, a Singular Value Decomposition is applied, i.e. the matrix $A$ is decomposed through $A=U S V^{T}$, where $U$ and $V$ are orthogonal matrices and $S$ is diagonal. The inversion of (6) now reads

$$
x_{i}=V_{i j} \frac{1}{S_{j j}} U_{j k}^{T} b_{k} .
$$

Small diagonal elements $S_{j j}$ may give rise to meaningless fluctuations in the solution distribution $x$. In order to suppress them, a regularization parameter $\xi$ which performs a smooth cutoff is introduced in (6), transforming the system of linear equations into the following minimization problem:

$$
|A x-b|^{2}+\xi|C x|^{2}=\min .
$$



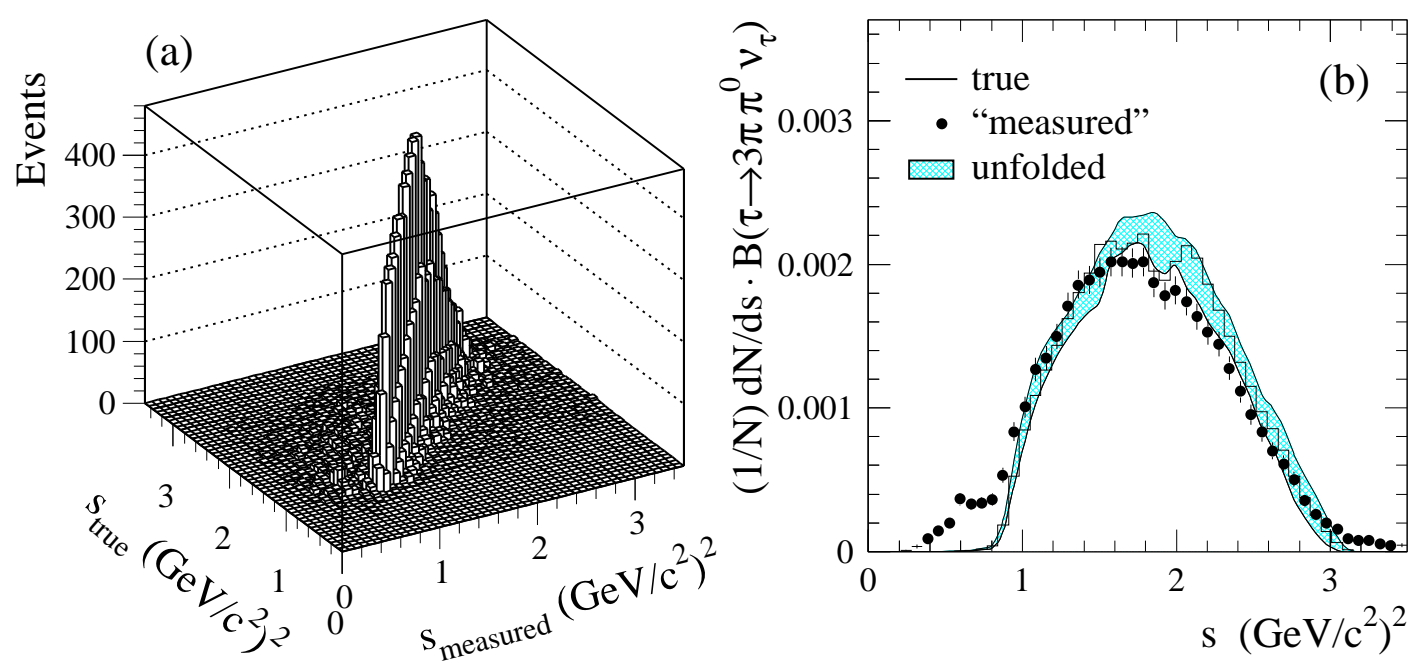

Figure 1: Plot (a) shows the detector response matrix used for the unfolding of the masssquared (s) spectrum of the decay $\tau^{-} \rightarrow 2 \pi^{-} \pi^{+} \pi^{0} \nu_{\tau}$. The true, reconstructed ("measured") and unfolded distributions of the corresponding Monte Carlo test spectrum are plotted in (b). The shaded region illustrates the uncertainty after unfolding, taking into account the statistical errors.

The additional regularization term $|C x|^{2}$ in (8) is the total curvature (sum of the squares of the second derivatives) of the solution distribution $x$, where $C$ is the symmetric curvature matrix defined as $C_{1,1}=C_{n, n}=-1, C_{i, i}=-2(i=2, \ldots, n-1)$, $C_{j, j+1}=C_{j+1, j}=1(j=1, \ldots, n-1)$ and $C_{i, j}=0$ otherwise. The cut parameter $\xi$ controls the relative importance of the two terms in Eq. (8): if $\xi$ is chosen too small, the solution $x$ contains meaningless fluctuations; on the other hand, if $\xi$ is too big, significant physical information is lost. The solution of the unfolding problem is now transformed into the optimal choice of $\xi$. In practice, the best $\xi$ is found by means of the parallel unfolding of a simulated test distribution $b_{\text {test }}$, for which the solution $x_{\text {test }}$ is known (see Fig. 1). The best choice of $\xi$ yields the smallest $\chi^{2}$ between the unfolded test distribution and the original true one. In order to make sure that this procedure applied to a simulated distribution leads to the optimal $\xi$ for data use, a test distribution has to be found, which reproduces the data as well as possible. For example, in the particular case of the $\tau^{-} \rightarrow 2 \pi^{-} \pi^{+} \pi^{0} \nu_{\tau}$ channel (see Fig. 3), the Monte Carlo simulation disagrees with the data in the peak region. As an appropriate test function, the unfolded data distribution is taken, found by an iterative adjustment of $\xi$ for the data. In this process it is important to distinguish significant information in the raw data distribution from insignificant statistical fluctuations, taking into account the mass resolution. Local statistical fluctuations in the measured distribution are washed out after unfolding. The systematic uncertainty coming from possible ambiguities in the choice of $\xi$ is described in Section 6.3.

In order to measure exclusive spectral functions, individual unfolding procedures with specific detector response matrices $A_{X}$ and cut parameters $\xi_{X}$ are applied for each $\tau$ decay 
channel $X$ considered.

\subsection{Spectral Functions for Exclusive $\tau$ Decay Modes}

The following vector $\tau$ decay modes are exclusively reconstructed: $\pi^{-} \pi^{0} \nu_{\tau}, \pi^{-} 3 \pi^{0} \nu_{\tau}$, $2 \pi^{-} \pi^{+} \pi^{0} \nu_{\tau}, 2 \pi^{-} \pi^{+} 3 \pi^{0} \nu_{\tau}$ and $3 \pi^{-} 2 \pi^{+} \pi^{0} \nu_{\tau}$. The measured mass-squared spectra corresponding to these channels are shown in Fig. 2 and 3. Before unfolding them, the $\tau$ and non- $\tau$ background and the strange contributions are subtracted using the

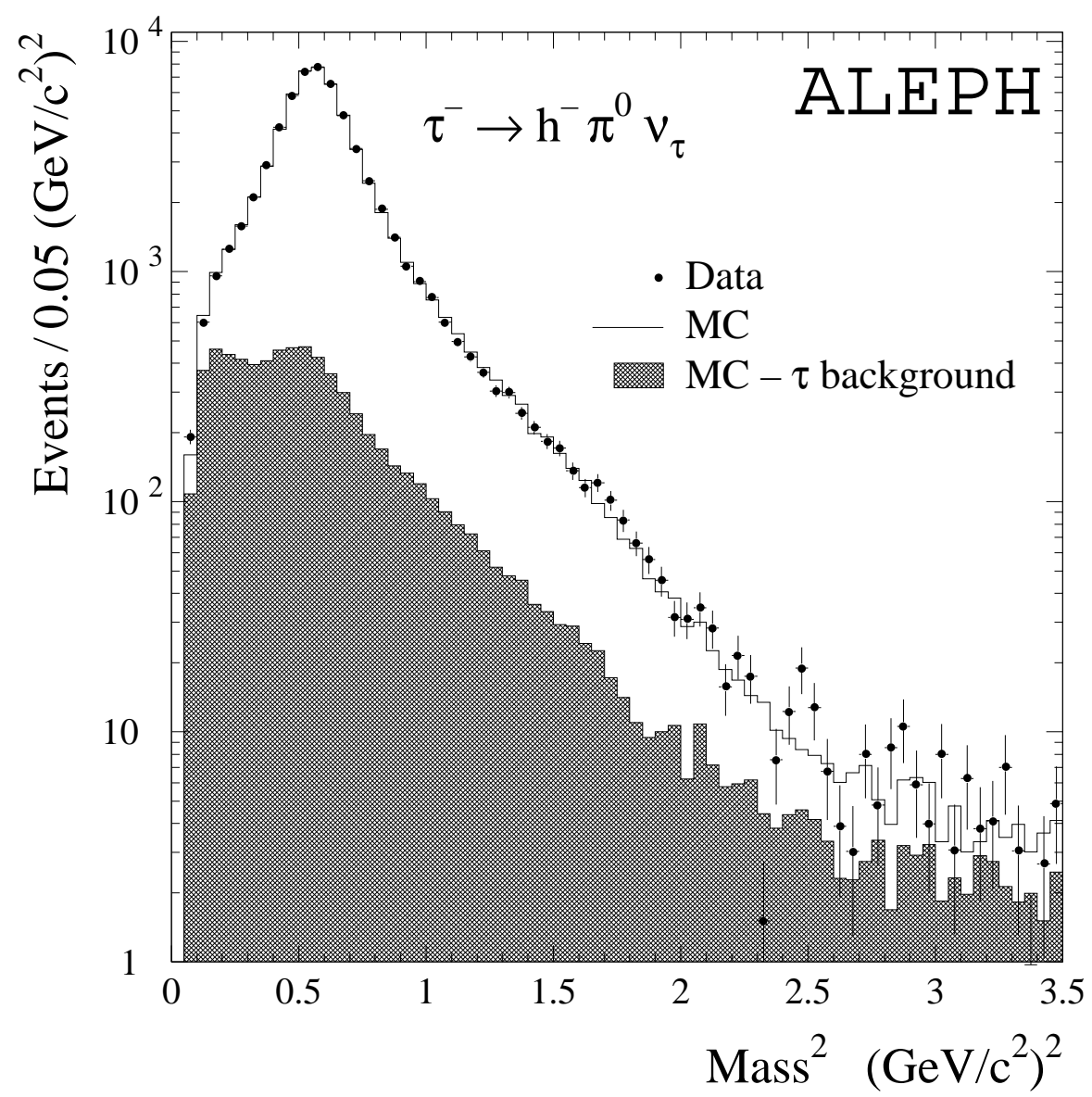

Figure 2: Invariant mass-squared distribution of the $\tau^{-} \rightarrow \mathrm{h}^{-} \pi^{0} \nu_{\tau}$ decay. The PDG [8] values $m_{\pi^{ \pm}}\left(m_{\pi^{0}}\right)$ are assumed for the masses of charged (neutral) particles.

Monte Carlo simulation ${ }^{3}$ which is based on models of resonance production implemented in KORALZ3. 8 with TAUOLA1. 5 as $\tau$ decay library $[15,16,17]$

The spectral functions of the dominant two- and four-pion modes are shown in the first three plots of Fig. 4. The errors shown are the diagonal elements of the covariance matrix. They include both statistical and systematic uncertainties. The $2 \pi^{-} \pi^{+} \pi^{0} \nu_{\tau}$ decay mode is compared to data of the ARGUS Collaboration [4].

\footnotetext{
${ }^{3}$ No strange contribution is assumed in the $2 \mathrm{~h}^{-} \mathrm{h}^{+} 3 \pi^{0} \nu_{\tau}$ channel.
} 

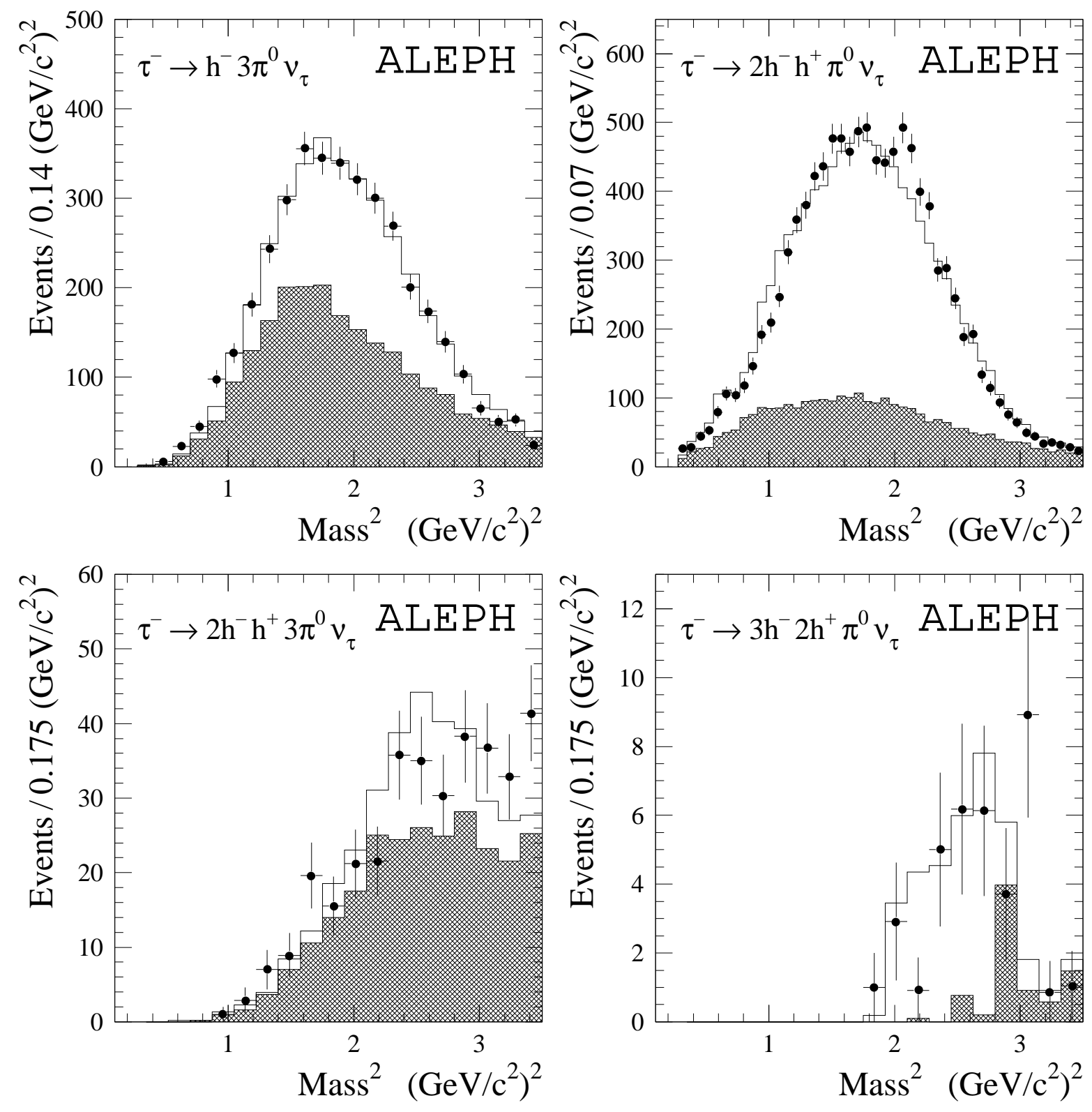

Figure 3: Invariant mass-squared distributions of the $\mathrm{h}^{-} 3 \pi^{0} \nu_{\tau}, 2 \mathrm{~h}^{-} \mathrm{h}^{+} \pi^{0} \nu_{\tau}, 2 \mathrm{~h}^{-} \mathrm{h}^{+} 3 \pi^{0} \nu_{\tau}$ and $3 \mathrm{~h}^{-} 2 \mathrm{~h}^{+} \pi^{0} \nu_{\tau}$ decay channels, respectively. The points are the measured data, the histograms represent the simulation and the hatched areas are the expected $\tau$ background distributions according to the simulation. 

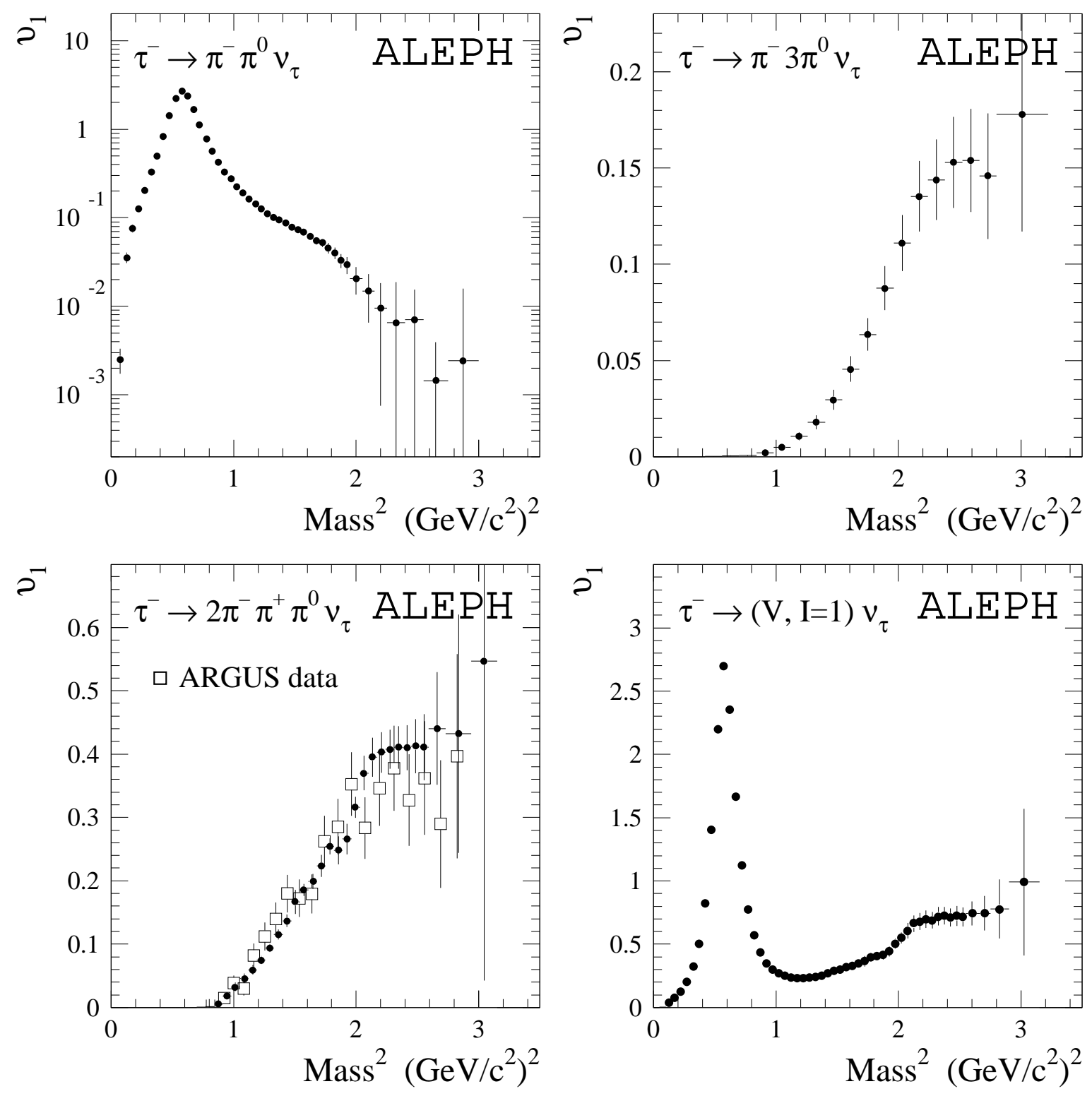

Figure 4: Spectral functions of the $\tau$ decay channels $\pi^{-} \pi^{0} \nu_{\tau}, \pi^{-} 3 \pi^{0} \nu_{\tau}, 2 \pi^{-} \pi^{+} \pi^{0} \nu_{\tau}$ and the total $\tau$ vector spectral function. The error bars are the diagonal elements of the covariance matrices. They contain both statistical and systematic contributions. The ARGUS data [4] in the $\tau^{-} \rightarrow 2 \pi^{-} \pi^{+} \pi^{0} \nu_{\tau}$ channels contain statistical errors only. 


\subsection{The Total $\tau$ Vector Spectral Function}

The total vector current spectral function is obtained by summing up the exclusive spectral functions with the addition of small contributions from unmeasured modes, as discussed below. Table 1 gives a survey of the exclusive decay modes considered, their classification and the corresponding branching ratios. If not otherwise specified, the latter were taken from ALEPH publications [21, 6] complemented by CLEO measurements [24, 25, 26, 27] and new results about branching fractions of $\tau$ decay modes involving kaons presented in Refs. [28, 29]. The individual fractions have been refitted so that the sum of all branching ratios adds up to $100 \%$. The branching ratios of the subsequent meson decays are taken from [8]. The small contributions labeled "MC" are taken from the Monte Carlo simulation. The two-, four- and six-pion modes are exclusively reconstructed as explained in Section 5.2. Special care is taken with isospin-violating $\omega$ and $\eta$ decays, and with final state Kaon production, as explained in the following:

- The decay channel $\tau^{-} \rightarrow \pi^{-} \omega \nu_{\tau}$ is partly reconstructed in the $2 \mathrm{~h}^{-} \mathrm{h}^{+} \pi^{0} \nu_{\tau}$ class $\left(\omega \rightarrow \pi^{+} \pi^{-} \pi^{0}\right)$, in the $\mathrm{h}^{-} 2 \pi^{0} \nu_{\tau}$ class $\left(\omega \rightarrow \pi^{0} \gamma\right)$ and in the $2 \mathrm{~h}^{-} \mathrm{h}^{+} \nu_{\tau}$ class $\left(\omega \rightarrow \pi^{+} \pi^{-}\right)$. Corrections to the total $\tau$ vector spectral function are applied for the latter two cases using invariant mass-squared distributions predicted by the Monte Carlo simulation. The systematic error due to the uncertainties in the Monte Carlo predictions is estimated to be $20 \%$ in every simulated mass bin. For all the following channels where the Monte Carlo simulation is used to complete the total $\tau$ vector current spectral function, the uncertainty is assumed to be $50 \%$ in every simulated mass bin in order to take into account the poorer knowledge of the spectrum.

- The decay channel $\mathrm{K}^{-} \mathrm{K}^{0} \nu_{\tau}$ consists of $50 \% \mathrm{~K}_{\mathrm{L}}^{0}$ and $50 \% \mathrm{~K}_{\mathrm{S}}^{0}$. The long-lived $\mathrm{K}_{\mathrm{L}}^{0}$ does not decay within the reach of the ALEPH tracking system. Its characteristic signature in the detector is a large energy deposition in the HCAL, exceeding the expected amount from the charged kaon alone. The decay rate for $\tau^{-} \rightarrow \mathrm{K}^{-} \mathrm{K}_{\mathrm{L}}^{0} \nu_{\tau}$ was measured by ALEPH $[30,31]$. The $\mathrm{K}^{-} \mathrm{K}_{\mathrm{L}}^{0} \nu_{\tau}$ decay is included in the inclusive $\tau^{-} \rightarrow \mathrm{h}^{-} \nu_{\tau}$ selection sample. The $\mathrm{K}^{-} \mathrm{K}_{\mathrm{S}}^{0} \nu_{\tau}$ decay is reconstructed in the $2 \mathrm{~h}^{-} \mathrm{h}^{+} \nu_{\tau}$ and $\mathrm{h}^{-} 2 \pi^{0} \nu_{\tau}$ samples which are dominated by axial-vector decays. Thus both $\mathrm{K}^{-} \mathrm{K}^{0} \nu_{\tau}$ contributions are taken from the simulation and added to the vector spectral functions.

- The $\tau$ decay channel $\pi^{-} \pi^{0} \eta \nu_{\tau}$ is reconstructed in the inclusive channels $\mathrm{h}^{-} 3 \pi^{0} \nu_{\tau}$, $\mathrm{h}^{-} 4 \pi^{0} \nu_{\tau}$ and $2 \mathrm{~h}^{-} \mathrm{h}^{+} 2 \pi^{0} \nu_{\tau}$. The last two contributions to the $\tau$ vector spectral functions are taken from the simulation, whereas in the $\mathrm{h}^{-} 3 \pi^{0} \nu_{\tau}$ channel, the measurement includes the contribution from the $\pi^{-} \pi^{0} \eta \nu_{\tau}$ mode.

- By virtue of isospin constraints it was deduced in Ref. [32] that the $\tau$ decay modes $\mathrm{K}^{-} \mathrm{K}^{0} \pi^{0} \nu_{\tau}, \mathrm{K}^{-} \mathrm{K}^{+} \pi^{-} \nu_{\tau}$ and $\mathrm{K}^{0} \overline{\mathrm{K}}^{0} \pi^{-} \nu_{\tau}$ are $\left(78_{-28}^{+22}\right) \%$ vector currents. Their contributions to the $\tau$ vector spectral functions are taken from the simulation. Neglecting so-called second class currents, the branching ratios of both $\mathrm{K} \overline{\mathrm{K}} \pi^{-}$states are equal using isospin symmetry ([33] and references therein).

- The $\tau$ decay into $\mathrm{K} \overline{\mathrm{K}} \pi \pi$ is poorly known. According to their respective final states, about $40 \%$ of the $\mathrm{K} \overline{\mathrm{K}} \pi \pi$ decays are reconstructed in vector channels while about 
$30 \%(30 \%)$ are selected in the axial-vector (strange) channels. The vector part of the total $\mathrm{K} \overline{\mathrm{K}} \pi \pi$ branching ratio is estimated to be $(0.08 \pm 0.08) \%$.

\begin{tabular}{|c|c|c|c|}
\hline $\begin{array}{l}\text { original } \tau^{-} \\
\text {vector mode }\end{array}$ & final state & input data & $\begin{array}{l}\tau \text { branching } \\
\text { ratio (in } \% \text { ) }\end{array}$ \\
\hline \multicolumn{2}{|c|}{$\overline{\pi^{-} \pi^{0} \nu_{\tau}}$} & $\mathrm{h}^{-} \pi^{0}$ & $25.35 \pm 0.19$ \\
\hline \multicolumn{2}{|c|}{$\pi^{-} 3 \pi^{0} \nu_{\tau}$} & $\mathrm{h}^{-} 3 \pi^{0}$ & $1.17 \pm 0.14$ \\
\hline \multicolumn{2}{|c|}{$2 \pi^{-} \pi^{+} \pi^{0} \nu_{\tau}$} & $2 \mathrm{~h}^{-} \mathrm{h}^{+} \pi^{0}$ & $2.54 \pm 0.09$ \\
\hline \multicolumn{2}{|c|}{$\begin{array}{c}2 \pi^{-} \pi^{+} 3 \pi^{0} \nu_{\tau} \\
3 \pi^{-} 2 \pi^{+} \pi^{0} \nu_{\tau} \\
\pi^{-} 5 \pi^{0} \nu_{\tau}\end{array}$} & $\begin{array}{c}2 \mathrm{~h}^{-} \mathrm{h}^{+} 3 \pi^{0} \\
3 \mathrm{~h}^{-} 2 \mathrm{~h}^{+} \pi^{0} \\
-\end{array}$ & $0.037 \pm 0.022^{(1)}$ \\
\hline$\pi^{-} \omega \nu_{\tau}$ & $\begin{array}{c}2 \pi^{-} \pi^{+} \pi^{0} \nu_{\tau} \\
\pi^{-} \pi^{0} \gamma \nu_{\tau} \\
2 \pi^{-} \pi^{+} \nu_{\tau}\end{array}$ & $\begin{array}{c}2 \mathrm{~h}^{-} \mathrm{h}^{+} \pi^{0} \\
\mathrm{MC} \\
\mathrm{MC}\end{array}$ & $\begin{array}{c}1.63 \pm 0.08 \\
0.155 \pm 0.010 \\
0.038 \pm 0.005\end{array}$ \\
\hline$\pi^{-} \pi^{0} \eta \nu_{\tau}$ & $\begin{array}{c}\pi^{-} \pi^{0} 2 \gamma \nu_{\tau} \\
\pi^{-} 4 \pi^{0} \nu_{\tau} \\
2 \pi^{-} \pi^{+} 2 \pi^{0} \nu_{\tau} \\
2 \pi^{-} \pi^{+} \pi^{0} \gamma \nu_{\tau}\end{array}$ & $\begin{array}{c}\mathrm{h}^{-} 3 \pi^{0} \\
\mathrm{MC} \\
\mathrm{MC} \\
\mathrm{MC} \\
\end{array}$ & $\begin{array}{l}0.068 \pm 0.011 \\
0.055 \pm 0.009 \\
0.039 \pm 0.007 \\
0.008 \pm 0.001\end{array}$ \\
\hline \multicolumn{2}{|c|}{$\mathrm{K}^{-} \mathrm{K}^{0} \nu_{\tau}$} & $\mathrm{MC}$ & $0.194 \pm 0.042$ \\
\hline \multicolumn{2}{|c|}{$\overline{\mathrm{K}^{-}} \mathrm{K}^{0} \pi^{0} \nu_{\tau}$} & $\mathrm{MC}$ & $0.100 \pm 0.046$ \\
\hline \multicolumn{2}{|c|}{$\begin{array}{c}\mathrm{K}^{-} \mathrm{K}^{+} \pi^{-} \nu_{\tau}[34,35] \\
\mathrm{K}^{0} \overline{\mathrm{K}}^{0} \pi^{-} \nu_{\tau}\end{array}$} & $\begin{array}{l}\mathrm{MC} \\
\mathrm{MC}\end{array}$ & $\begin{array}{c}0.168 \pm 0.030 \\
0.168 \pm 0.030^{(1)}\end{array}$ \\
\hline \multicolumn{2}{|c|}{$\mathrm{KK} \pi \pi$ Vector } & $\mathrm{MC}$ & $0.08 \pm 0.08$ \\
\hline \multicolumn{3}{|c|}{ Total Vector } & $31.71 \pm 0.31$ \\
\hline
\end{tabular}

${ }^{1}$ The branching ratio is obtained using isospin invariance as explained in the text.

Table 1: Tau decays contributing to the total vector current spectral function. The first and second columns contain the physical decay modes and the corresponding final states. The third column shows the topology as reconstructed in the detector. Contributions from the "MC" labeled modes, are taken from the Monte Carlo simulation. The right-hand column gives the corresponding final state branching ratios. The vector part of the $\mathrm{K} \overline{\mathrm{K}} \pi \nu_{\tau}$ modes is estimated to be $\left(78_{-28}^{+22}\right) \%$ [32]. The last line gives the total branching fraction of vector hadronic $\tau$ decays.

- For the six-pion final states, one can deduce most restrictive upper bounds for unknown or unprecisely measured channels utilizing isospin invariance in conjunction with the method developed by Pais [36] (see Appendix) in which the $\tau$ partial width is decomposed into a set of orthogonal classes $\{i j k\}$. As values for the corresponding branching ratios one may take half the bounds with $100 \%$ uncertainty. Using advantageously only the $3 \pi^{-} 2 \pi^{+} \pi^{0} \nu_{\tau}$ data $[21,8,24]$ and subtracting from it the contribution from the isospin violating, axial-vector $2 \pi^{-} \pi^{+} \eta$ final state [37], we 


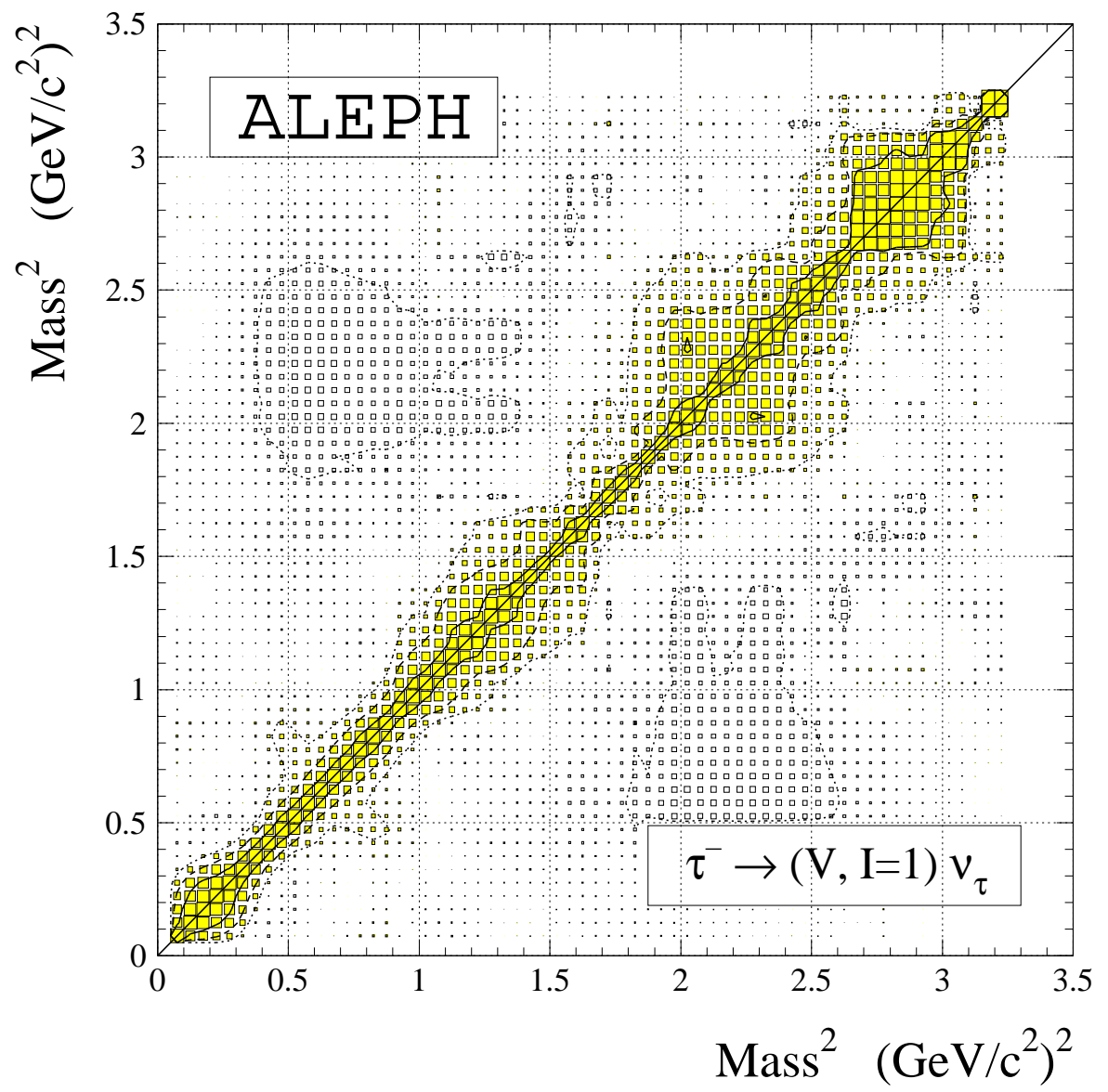

Figure 5: Correlations between the data points of the total $\tau$ vector spectral function shown in Figure 4. The shaded (empty) boxes are proportional to the positive (negative) correlation in the interval $[0,1]([0,|-1|])$. The contour lines illustrate the $75 \%$ (solid line), $50 \%$ (dashed line) and $25 \%$ (dotted line) correlations, respectively, for positive and negative correlations.

obtain a total vector six-pion branching ratio of $B\left(\tau^{-} \rightarrow(6 \pi)_{V}^{-} \nu_{\tau}\right)=(0.030 \pm 0.030) \%$ from an upper (lower) limit when choosing the class $\{330\}(\{411\})$ to be dominant.

The total $\tau$ vector spectral function is shown in Fig. 4, while Fig. 5 depicts the corresponding correlation matrix.

\section{$6 \quad$ Systematic Errors}

The study of systematic errors affecting the measurement is subdivided into several classes according to their origin, i.e., the photon and $\pi^{0}$ reconstruction, the charged track measurement, the unfolding procedure and additional sources. All systematic uncertainties concerning the classification are contained in the errors of the branching ratios measured under identical conditions using the same analysis techniques [21]. Only the systematic effects affecting the mass-squared distributions need to be examined here. 


\subsection{The $\pi^{0}$ Reconstruction}

The following effects are studied:

- The photon energy calibration is performed by comparing the ratio of the ECAL cluster energy of electrons, reconstructed as if they are photons, to the momentum of the track in data and simulation. The electrons are taken from two-photon processes at low energies, $\tau^{-} \rightarrow e^{-} \bar{\nu}_{e} \nu_{\tau}$ decays at low and intermediate energies, and Bhabha events at beam energy. At the lowest energies (below $10 \mathrm{GeV}$ ) electron showers cannot be used because of the large curvature of their trajectories. To circumvent this, neutral pion decays with wide opening angles are used for the energy calibration. Special care is necessary to correct the calibration for energy dependent effects, i.e., differences in the distribution of the photon opening angle between data and the simulation due to an excess of fake photons in the data. Depending on the polar angle, the final relative calibration uncertainty is found to be about $0.6-2.3 \%$ for energies up to $3 \mathrm{GeV}, 0.4-2.2 \%$ between 3 and $10 \mathrm{GeV}$, $0.5-1 \%$ between 10 and $20 \mathrm{GeV}, 0.3-0.5 \%$ between 20 and $40 \mathrm{GeV}$ and finally about $0.2 \%$ at beam energy. The corresponding systematic error is determined by varying the energy dependent calibration within its respective uncertainty. It should be
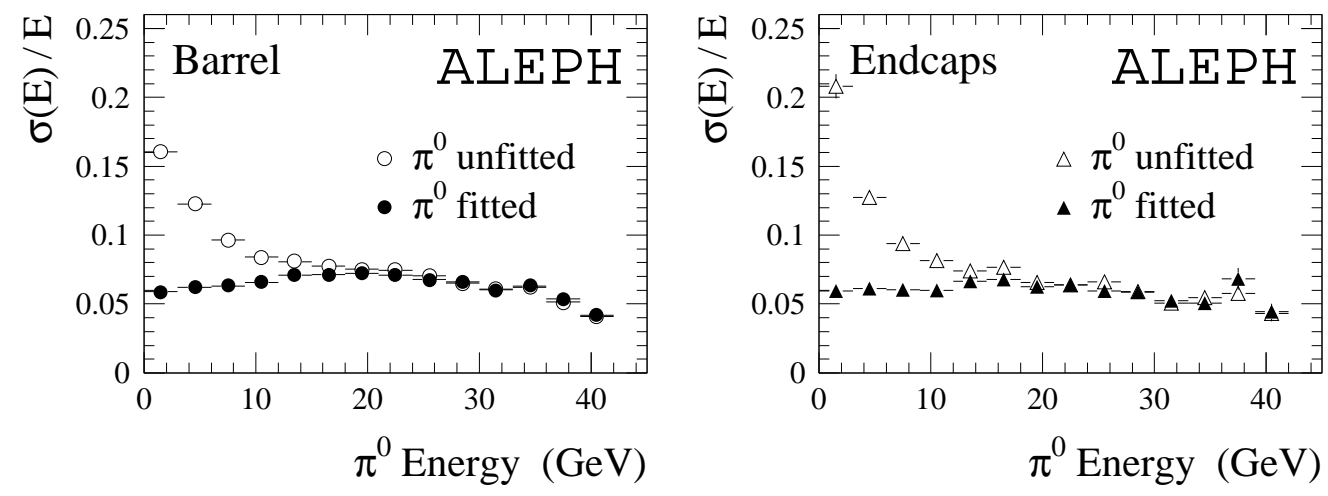

Figure 6: Energy resolution of resolved and unresolved $\pi^{0}$ 's in the simulation as a function of the $\pi^{0}$ energy before and after the $\pi^{0}$ mass constrained fit in the barrel $(|\cos \Theta| \leq 0.774)$ and endcaps $(0.774<|\cos \Theta| \leq 0.95)$, respectively.

emphasized that due to a precise measurement of the two photon opening angle at low energies, uncertainties in the calibration are highly recovered by the $\pi^{0}$ mass constrained fit. This is not valid at high energies. Fig. 6 shows the gain achieved in the energy resolution when performing the fit, as found in the simulation.

- The ECAL energy resolution in data and simulation at high energies is studied using Bhabha events with low radiation $\left(E_{\mathrm{ECAL}} / E_{\mathrm{TPC}} \simeq 1\right.$, at beam energy). It is found to be overestimated in the simulation by about $14 \%$. At low energies, the resolution of photon energies is directly tested, using the experimental width of the reconstructed $\pi^{0}$ mass. All detected deviations in the energy resolution are corrected in the simulation, while its uncertainties are taken as systematic errors. 

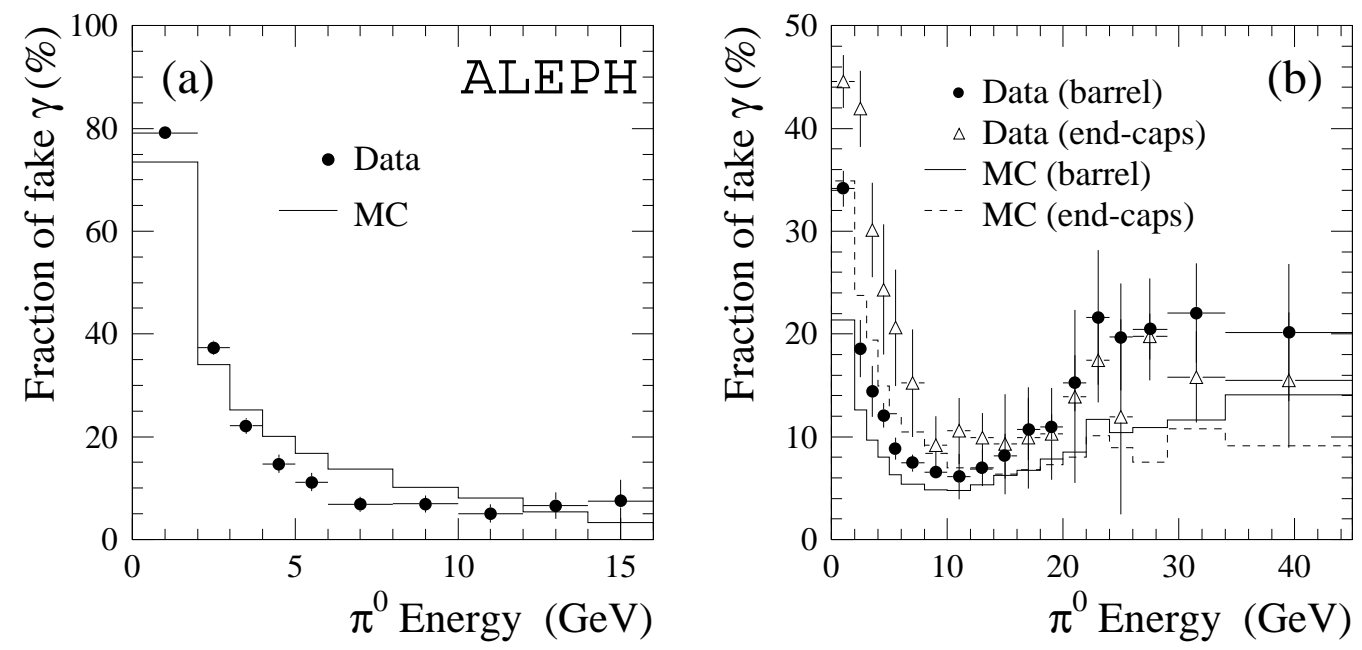

Figure 7: Fraction of fake photons in (a) residual $\pi^{0}$ 's and (b) resolved $\pi^{0}$ 's in hadronic $\tau$ decay channels as a function of the $\pi^{0}$ energy.

- The reference distributions for the likelihood procedure to evaluate the photon probability are obtained from the Monte Carlo simulation. These distributions are slightly corrected after detailed comparisons between data and Monte Carlo simulation. By switching off this correction, a conservative systematic error is determined (see Ref. [21] for more details).

- The threshold for photon detection is $300 \mathrm{MeV}$. The comparison of low energy photons belonging to resolved $\pi^{0}$ 's in data and Monte Carlo simulation shows that the inefficiency in data is larger by $(4.4 \pm 3.4) \%$ with respect to the simulation [21]. This is corrected in the simulation. A variation of the threshold by $\pm 20 \mathrm{MeV}$ corresponds to a change of the photon reconstruction efficiency near threshold of $3.4 \%$. A variation of the photon energy threshold by $\pm 30 \mathrm{MeV}$ is used to extract a conservative systematic error due to the quoted uncertainty in the determination of the efficiency.

- A cut on the minimal distance between the barycentre of an electromagnetic cluster and the closest track is applied in order to veto fake photon candidates from hadronic interactions in the ECAL. Thus, a cluster deposited in the ECAL is considered as a photon candidate if its minimal distance to the nearest charged track exceeds $2 \mathrm{~cm}$. The comparison of the distribution of this distance between data and simulation below $8 \mathrm{~cm}$ shows good agreement. Similar to the minimal photon energy threshold, a possible discrepancy can be covered by a variation of the minimal distance cut value by $\pm 0.1 \mathrm{~cm}$, which is used to extract the corresponding systematic error (see Ref. [21] for more details).

- The data suffer from an excess of fake photons compared to the simulation. This excess is measured by fitting the simulated distributions of the $\pi^{0}$ probabilities for resolved $\pi^{0}$ 's and the single photon probabilities for residual photons originating 

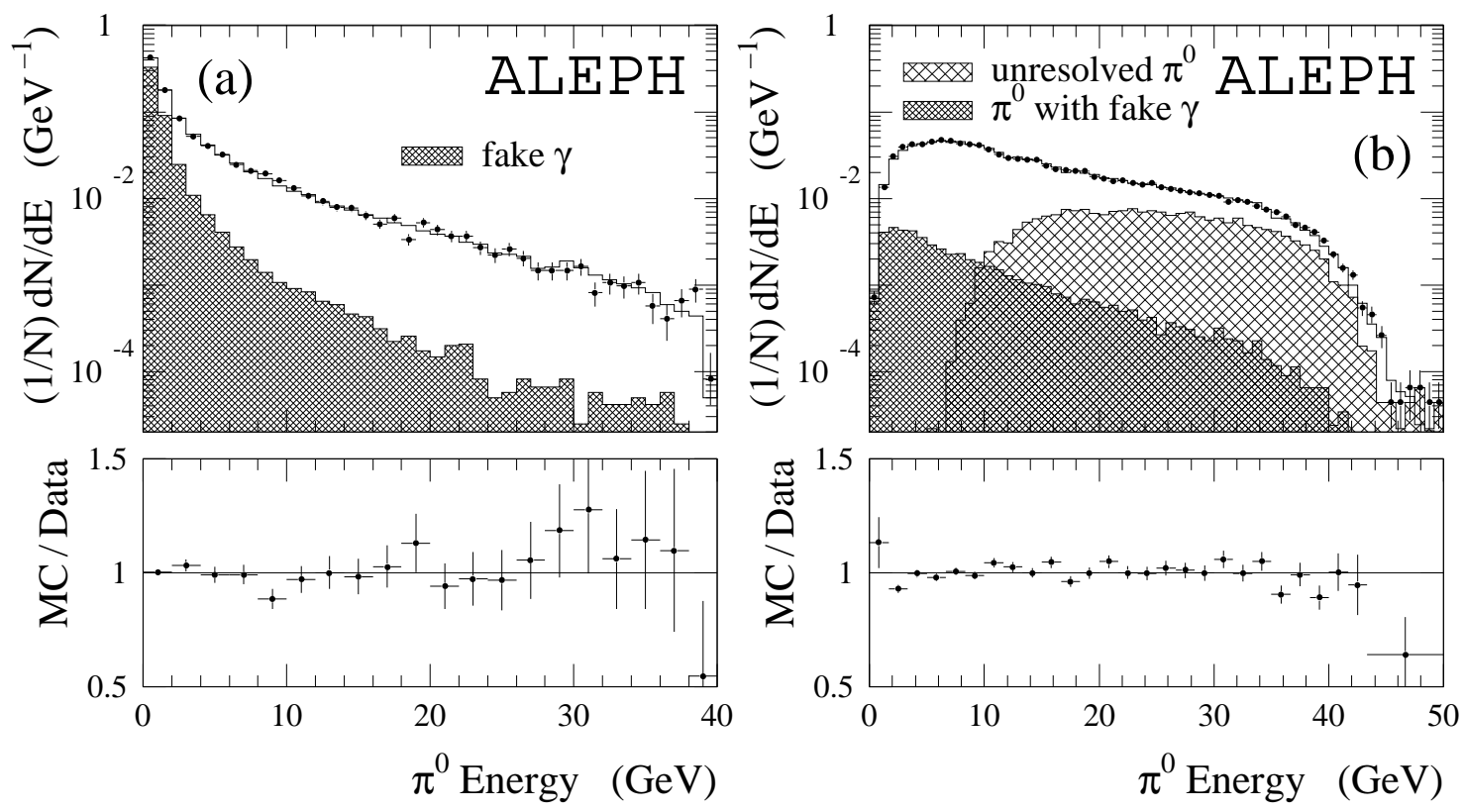

Figure 8: Spectra of $\pi^{0}$ energy for (a) residual $\pi^{0}$ 's and (b) reconstructed $\pi^{0}$ 's in $\tau^{-} \rightarrow \mathrm{h}^{-} \pi^{0} \nu_{\tau}$ decays for data (points) and the Monte Carlo simulation (histogram). The shaded areas illustrate the fake photon contamination. The unresolved component of the $\pi^{0}$ sample in (b) is shown by the hatched area. The lower plots show the corresponding Monte Carlo/data ratios.

from fake and good photons to the data. Fig. 7 shows the fraction of fake photons in the residual and resolved $\pi^{0}$ sample of hadronic $\tau$ decays as a function of the $\pi^{0}$ energy. Unresolved $\pi^{0}$ 's occur preferentially at high energy. They have a negligible contamination of fake photons. The enhancement of the fraction of fake photons in the high energy region in the resolved $\pi^{0}$ sample is due to split-off effects when energy fluctuations produce a low energy satellite cluster, misidentified as a photon, near the unresolved one. The measured spectrum of invariant mass of each considered decay channel is corrected according to its corresponding energydependent excess of fake photons. The statistical errors of the probability fits together with the systematic uncertainty coming from genuine differences in the probability distributions (extracted by switching off the corrections of the respective likelihood variable distributions as discussed in Ref. [21]) are used to estimate the corresponding systematic uncertainty.

- The apparent $\pi^{0}$ mass and resolution depend on the the $\pi^{0}$ energy. This dependence is used as a reference in order to calculate the probability for two photons to originate from a $\pi^{0}$. Varying these dependences within their uncertainties yields the corresponding systematic errors. In performing the constrained fit, the measured photon energies and their opening angle are refitted in order to constrain the apparent energy dependent masses to the nominal $\pi^{0}$ mass. 
Fig. 8 shows the energy spectrum of residual and reconstructed $\pi^{0}$ 's after applying the corrections mentioned above. The contamination of the residual sample with fake photons is illustrated by the shaded area (upper left plot).

\subsection{Systematics in the Measurement of Charged Tracks}

The following systematic effects are studied:

- The momentum calibration is performed using muon pairs and final states originating from narrow resonances. The corresponding systematic errors are found by varying the partly correlated errors of the polar angle dependent calibration. These fluctuations are assumed to be sagitta errors and therefore scale with the squared particle momentum. They amount up to an uncertainty of $0.1 \%$. The relative uncertainty on the magnetic field is estimated to be lower than $0.03 \%$ [38].

- The momentum resolution is studied with $\mu$ pair events at beam energy. A correction of about $20 \%$ has to be applied to the simulation. The uncertainty on this correction yields a systematic error.

Fig. 9a shows the momentum spectrum of the charged hadron in $\tau^{-} \rightarrow \mathrm{h}^{-} \pi^{0} \nu_{\tau}$ decays for data and the Monte Carlo simulation. Good agreement is found.
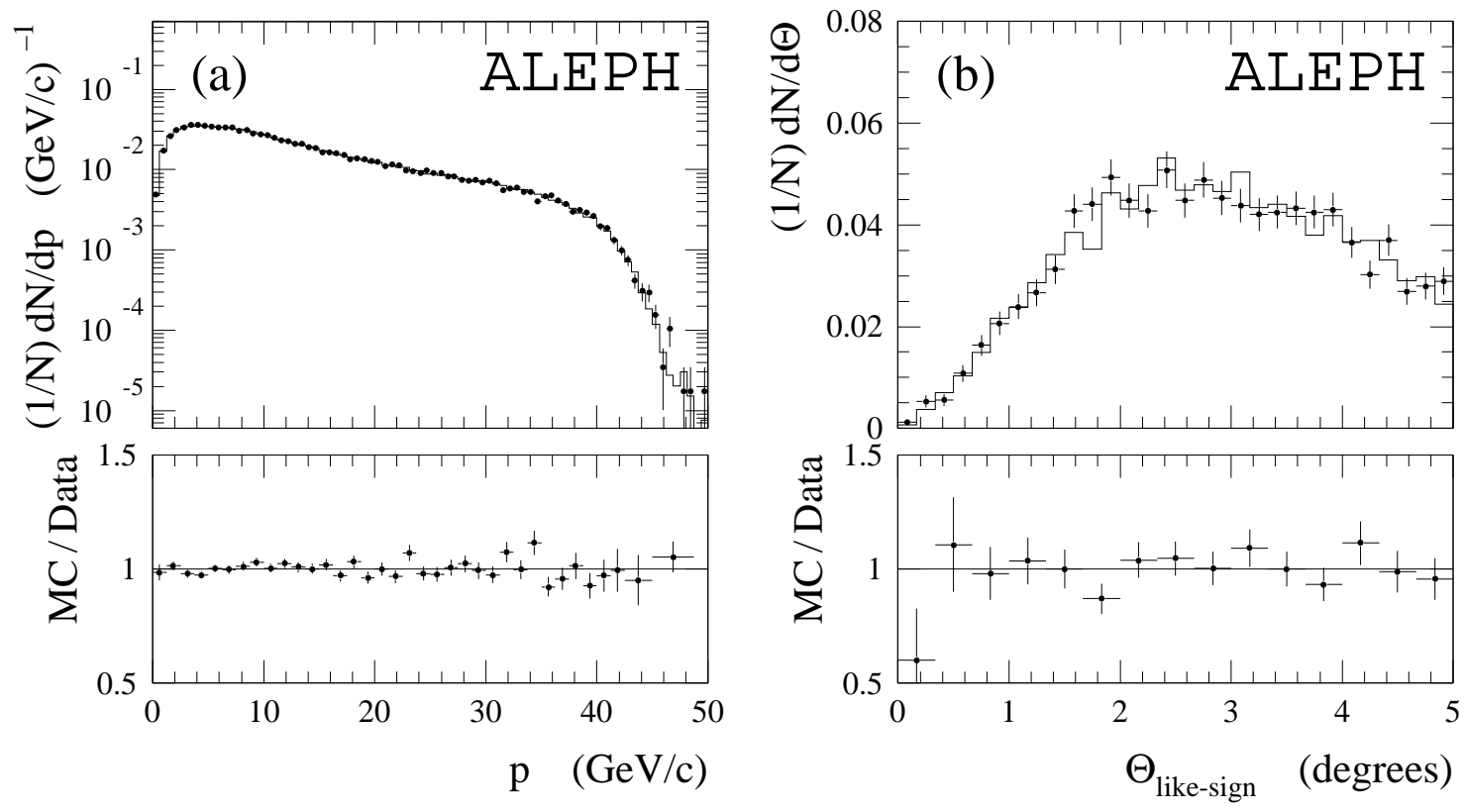

Figure 9: Momentum spectrum of the charged hadron in $\tau^{-} \rightarrow \mathrm{h}^{-} \pi^{0} \nu_{\tau}$ decays (a) and distribution of the angle between equal charged tracks in three-prong $\tau$ decays (b) for data (points) and the Monte Carlo simulation (histogram). The lower plots show the corresponding Monte Carlo/data ratios. 
- The reconstruction efficiency of highly collimated tracks as they occur in multiprong events can be tested by comparing the angular distribution between like-sign tracks in the data and the simulation in $\tau^{-} \rightarrow 2 \mathrm{~h}^{-} \mathrm{h}^{+} \nu_{\tau}$ events. They are found to be in good agreement (see Fig. $9 \mathrm{~b}$ ). The corresponding systematic uncertainty is negligible.

- The effect of secondary nuclear interactions is studied by comparing the invariant mass-squared distributions of enriched data samples to the Monte Carlo simulation. An enrichment of about $48 \%$ of events containing nuclear interactions is found in the simulation when requiring a minimal distance $d_{0}$ between $\operatorname{track}(\mathrm{s})$ and interaction point of at least $1 \mathrm{~cm}$ [13]. The resulting hadronic invariant mass spectra are found to be in good agreement between data and the simulation. Again, the systematic effect on the shape of the measured distributions is negligible. The consequences of topology-changing effects, i.e., a feed-through of events between different $\tau$ decay modes, are contained in the branching ratio uncertainty and are described in detail in Ref. [21].

\subsection{Systematic Errors in the Unfolding Procedure}

Two important tests are performed to evaluate potential systematic biases introduced by the unfolding procedure:

- The cut parameter $\boldsymbol{\xi}$ of Eq. (8) is varied in the region $\chi^{2} /$ dof $\leq 2$ around the minimum, obtained when unfolding the corresponding test distribution under same conditions as the data, i.e. using the same detector response matrix. The appropriate test distribution is designed to reproduce well the data (as explained in Section 5.1). The test distribution must not introduce additional statistical fluctuations and should therefore be smoothed as if it were built with infinite statistics. Nevertheless, the corresponding $\chi^{2}$ is computed within the accuracy of given data statistics.

- The total bin-to-bin differences between the unfolded test and its true distribution is considered as an additional systematic uncertainty of the unfolding procedure in order to be more conservative. It is taken as a diagonal, i.e., uncorrelated, systematic error in the unfolded data distribution (see Fig. 1).

\subsection{Other Sources of Systematic Errors}

In addition, the following sources are examined:

- The limited statistics in the simulation causes a systematic error which is determined by making the two-dimensional entries in the detector response matrices fluctuate independently.

- The uncertainties in the hadronic branching ratios introduce the dominant systematic errors in the subtraction of the $\tau$ background and kaon channels from data and in the respective normalization of the spectral functions. They are quoted 
by varying the branching ratios, taking into account the correlation matrix given in Ref. [21].

- The non- $\tau$ background is varied by $\pm 50 \%$.

All mentioned sources of systematic errors other than those originating from uncertainties in the branching ratios, i.e., the absolute normalization of the respective mass-squared distributions, are only considered when they concern the shape of the measured distribution. Their effect on the normalization is already included in the error of the corresponding branching ratio.

To illustrate the respective importance of the mentioned systematic uncertainties, one may perform an integration over the spectral function with some given kernel, characteristic of the physical problem to be studied. The integration error is then obtained by Gaussian error propagation, taking into account the correlations; using moderate, $s$-dependent integration kernels, the integration error will clearly be dominated by normalization uncertainties, i.e., the errors on the respective $\tau$ branching ratios. However, the error of an integration with strongly $s$-dependent weighting kernels enhancing the low energy part of the spectral functions will be dominated by systematics (mainly due to the fake photon rejection and the photon efficiency correction at threshold), while the central energy region $\left(0.6-1.4 \mathrm{GeV}^{2} / c^{4}\right)$ is statistically limited. When enhancing the higher part of the spectrum, the integration error will be equally dominated by uncertainties due to the unfolding process, and by limited data and Monte Carlo statistics.

\section{Applications}

Two applications of this analysis are described. In the first one the measured spectral functions of the two- and four-pion final states are compared via isospin rotation to data from $e^{+} e^{-}$annihilation experiments. The second application deals with a fit of the $\pi^{-} \pi^{0}$ spectral function in terms of vector resonances.

\subsection{Comparison to $e^{+} e^{-}$Results}

In this section, the most precise spectral function measurements of the $\tau$ vector current final states $\pi^{-} \pi^{0}, \pi^{-} 3 \pi^{0}$ and $2 \pi^{-} \pi^{+} \pi^{0}$ are compared to the cross sections of the corresponding $e^{+} e^{-}$annihilation isovector states $\pi^{+} \pi^{-}, \pi^{+} \pi^{-} \pi^{+} \pi^{-}$and $\pi^{+} \pi^{-} \pi^{0} \pi^{0}$. Using Eq. (1) and isospin rotation [39, 40] (see Appendix) the following relations hold:

$$
\begin{aligned}
\sigma_{e^{+} e^{-} \rightarrow \pi^{+} \pi^{-}}^{I=1,0}-\Delta I_{\rho \omega} & =\frac{4 \pi \alpha^{2}}{s} v_{1, \pi^{-} \pi^{0} \nu_{\tau}}, \\
\sigma_{e^{+} e^{-} \rightarrow \pi^{+} \pi^{-} \pi^{+} \pi^{-}}^{I=1} & =2 \cdot \frac{4 \pi \alpha^{2}}{s} v_{1, \pi^{-} 3 \pi^{0} \nu_{\tau}}, \\
\sigma_{e^{+} e^{-} \rightarrow \pi^{+} \pi^{-} \pi^{0} \pi^{0}}^{I=1} & =\frac{4 \pi \alpha^{2}}{s}\left[v_{1,2 \pi^{-} \pi^{+} \pi^{0} \nu_{\tau}}-v_{1, \pi^{-} 3 \pi^{0} \nu_{\tau}}\right] .
\end{aligned}
$$


In Eq. (9) the small isospin-violating, isoscalar, electromagnetic contribution $\omega(782) \rightarrow$ $\pi^{+} \pi^{-}$is taken into account through its interference with the main isovector contribution yielding the (s-dependent) correction $\Delta I_{\rho \omega}$ obtained from a fit of the total $e^{+} e^{-} \rightarrow \pi^{+} \pi^{-}$ cross section [2]. The mass and the width of the $\omega(782)$ are taken from Ref. [8].

The $e^{+} e^{-} \rightarrow \pi^{+} \pi^{-}$measurements are taken from OLYA [44, 45], TOF [46], NA7 [47], CMD [44], DM1 [48], DM2 [49], MEA [50] and BCF [51, 52]. The comparison to $\tau$ data according to Eq. (9) is shown in Fig. 10. The two sets of measurements are very precise and in good agreement. Fig. 10b shows the square of the isovector pion form factor $F_{\pi}^{I=1}$ in the threshold region of the two-pion production for $\tau$ and $e^{+} e^{-}$data. A second order expansion can be used as a description of $F_{\pi}^{I=1}$ at very low energies $[42,43]$ :

$$
F_{\pi}^{\mathrm{ChPT}} \simeq 1+\frac{1}{6}\left\langle r^{2}\right\rangle_{\pi} s+c_{\pi} s^{2}+O\left(s^{3}\right) .
$$

Exploiting precise results from space-like data [53], the pion charge radius-squared $\left\langle r^{2}\right\rangle_{\pi}=(0.431 \pm 0.026) \mathrm{fm}^{2}$ and the coefficient $c_{\pi}=(3.2 \pm 1.0) \mathrm{GeV}^{-4}$ from Eq. (12) have recently been determined by means of a simultaneous fit [54]. An expanded view of the $\rho(770)$ peak region is given in Fig. 10c.

The $e^{+} e^{-} \rightarrow \pi^{+} \pi^{-} \pi^{+} \pi^{-}$data are taken from OLYA [55], ND [56], MEA [57], CMD [58], DM1 [59, 60], DM2 [61, 62, 63] and M3N [64]. The comparison to the decay channel $\tau^{-} \rightarrow \pi^{-} 3 \pi^{0} \nu_{\tau}$ using Eq. (10) is shown in Fig. 11a. It is found to be in rather good agreement.

The $e^{+} e^{-} \rightarrow \pi^{+} \pi^{-} \pi^{0} \pi^{0}$ data are taken from OLYA [55], ND [56], M2N [65], DM2 [61, 62, 63] and M3N [64]. The measurements originating from different $e^{+} e^{-}$ experiments show some inconsistencies (see Fig. 11b). On the low mass side, the cross section is dominated by ND and OLYA data from the VEPP-2M storage ring at Novosibirsk. The ND measurement points are significantly higher than the OLYA data. At higher mass, data are dominated by the Orsay experiments DM2 and M3N: the DM2 cross section points are significantly lower than the M3N measurements. Tau data slightly favor the OLYA data on the low mass side; furthermore, they are clearly higher than the DM2 results in the central region between 2 and $2.6 \mathrm{GeV}^{2} / c^{4}$. The small dots in Fig. 11b illustrate the resonant $\omega \pi^{0} \rightarrow \pi^{+} \pi^{-} \pi^{0} \pi^{0}$ contribution taken from ND [56] and DM2 [62].

Finally, the total $e^{+} e^{-}$isovector cross section is compared to the $\tau$ vector current spectral function. The following contributions require some discussion:

- The isospin descriptions for the two- and four-pion final states are easily found by inverting Eqs. (9), (10) and (11). The isoscalar $\omega \rightarrow \pi^{+} \pi^{-}$contribution is subtracted from the total $\pi^{+} \pi^{-}$cross section.

- The four-pion final state of the $\omega \pi^{0}$ mode is already contained in the $\pi^{+} \pi^{-} \pi^{0} \pi^{0}$ cross section. A $11.2 \%$ correction for the other $\omega$ decay modes is applied.

- The $e^{+} e^{-} \rightarrow \pi^{+} \pi^{-} \eta$ data are taken from ND [56] and DM2 [66].

- The cross sections for the six-pion final states $3 \pi^{+} 3 \pi^{-}$and $2 \pi^{+} 2 \pi^{-} 2 \pi^{0}$ were measured by DM1 [67], M3N [64], CMD [58] and DM2 [68]. Using Pais' classes [36] 

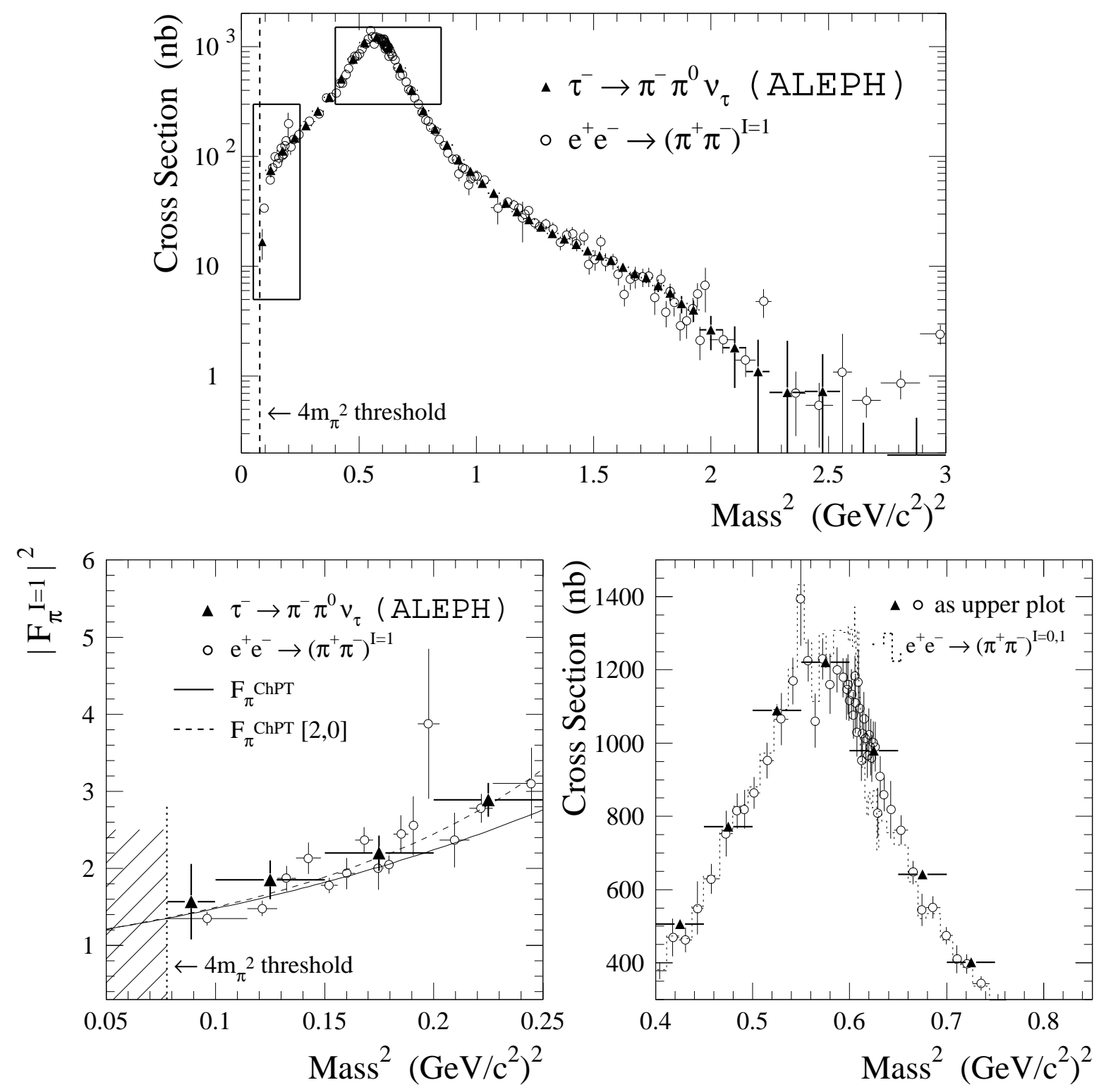

Figure 10: The two-pion data from $\tau$ decays compared to the corresponding isovector $e^{+} e^{-}$ cross section (data points from different $e^{+} e^{-}$experiments, measured at the same mass have been averaged). Both distributions are shown with statistical and systematic errors. The two rectangles indicate the regions that are expanded in (b) and (c). Figure (b) shows the pion form factor near threshold. The chiral expansion $F_{\pi}^{\mathrm{ChPT}}$ is defined in Eq. (12). The additional function labeled "[2, 0]" (indistinguishable from "[1,1]" in the plotted energy region) denotes different parametrizations (Padé approximants [41]) deduced from Chiral Perturbation Theory as discussed in Ref. [42, 43]. The dotted line in Figure (c) represents the total (uncorrected) isoscalar and isovector $e^{+} e^{-}$cross section. 

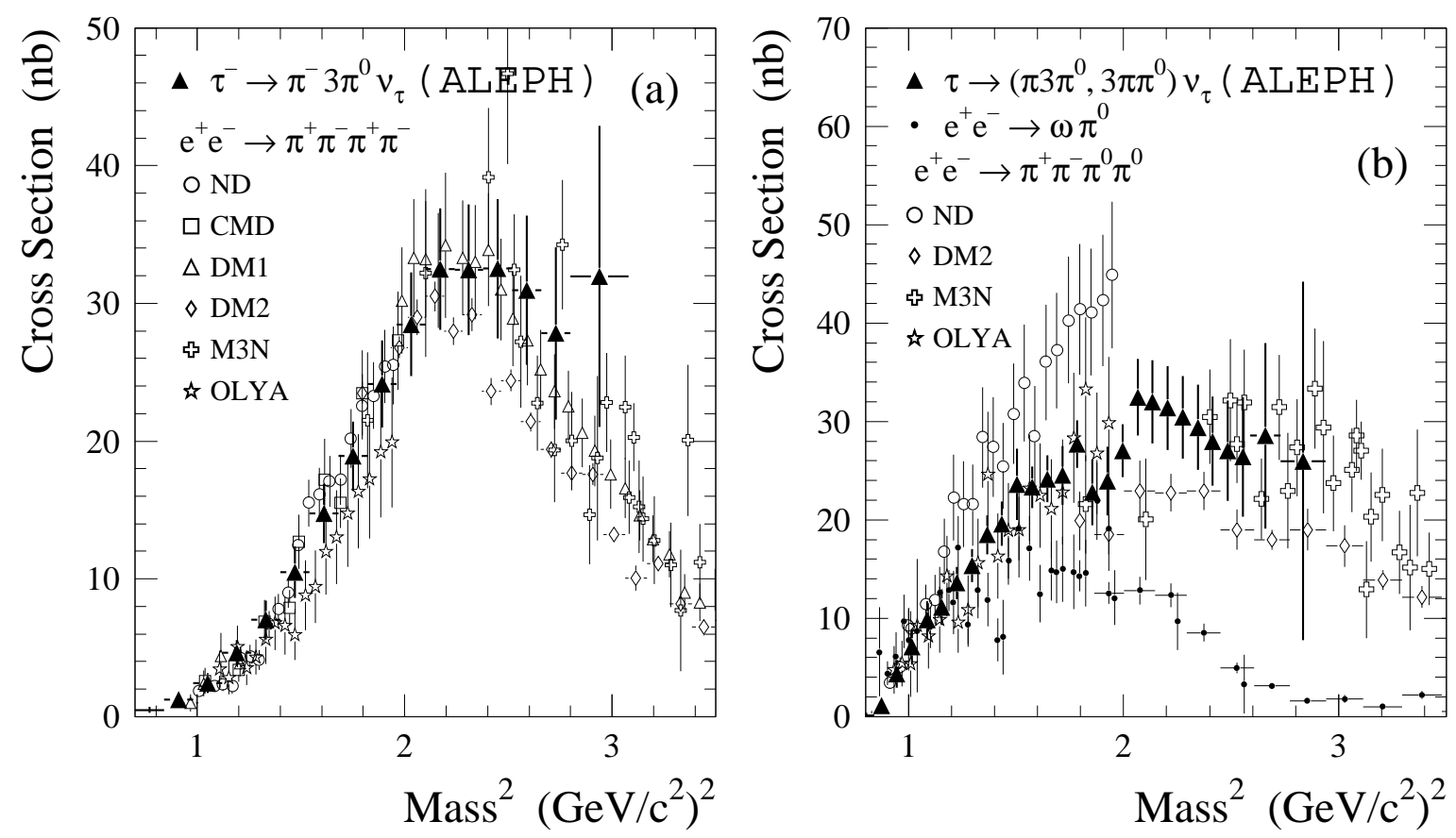

Figure 11: Comparison of the isospin-rotated four-pion $\tau$ data with the corresponding $e^{+} e^{-}$cross sections. The error bars shown contain both statistical and systematic errors. An enhancement in the low mass part of the $\pi^{+} \pi^{-} \pi^{0} \pi^{0}$ channel in (b) is expected from the resonant $\omega \pi$ contribution (small points).

(see Appendix) one can deduce an upper limit for the unknown $\sigma_{\pi^{-} \pi^{+} 4 \pi^{0}}$ cross section. Assuming conservatively the classes $\{411\}$ and $\{510\}$ to be dominant, one obtains $\sigma_{\pi^{+} \pi-4 \pi^{0}} \leq(3 / 2) \times \sigma_{2 \pi^{-} 2 \pi^{+} 2 \pi^{0}}-(9 / 24) \times \sigma_{3 \pi^{-} 3 \pi^{+}}$.

- To extract the isovector part of the $\mathrm{K}^{+} \mathrm{K}^{-}$and $\mathrm{K}_{\mathrm{S}}^{0} \mathrm{~K}_{\mathrm{L}}^{0}$ states, the $\mathrm{SU}(3)$ relation between pion and kaon form factors is adopted to infer the relation [52]

$$
\sigma_{\mathrm{K}^{+} \mathrm{K}^{-}}(s)=\frac{\beta_{\mathrm{K}}^{3}}{4 \beta_{\pi}^{3}} \sigma_{\pi^{+} \pi^{-}}(s)
$$

where $\beta_{\mathrm{K}, \pi}=\left(1-4 M_{\mathrm{K}, \pi}^{2} / s\right)^{1 / 2}$. This can be directly related to the $\tau^{-} \rightarrow \mathrm{K}^{-} \mathrm{K}^{0} \nu_{\tau}$ spectral function, for which, due to the uncertainty of the relation (13), a total systematic uncertainty of $25 \%$ is assumed.

- The DM1 and DM2 collaborations $[69,70]$ made some effort to isolate a small isovector component of the $e^{+} e^{-} \rightarrow \mathrm{K}^{ \pm} \mathrm{K}_{\mathrm{S}}^{0} \pi^{\mp}$ cross section. This can be scaled up to the full $\mathrm{KK} \pi$ contribution which can be related to the corresponding $\tau$ spectral function using isospin symmetry.

- The inclusive reaction $e^{+} e^{-} \rightarrow \mathrm{K}_{\mathrm{S}}^{0}+X$ was analyzed by DM1 [71]. Having subtracted from its cross section the separately measured contributions of the final states $\mathrm{K}_{\mathrm{S}}^{0} \mathrm{~K}_{\mathrm{L}}^{0}, \mathrm{~K}_{\mathrm{S}}^{0} \mathrm{~K}^{+} \pi^{-}$and $\mathrm{K}_{\mathrm{S}}^{0} \mathrm{~K}_{\mathrm{L}}^{0} \pi^{0}$, it still includes the modes $\mathrm{K}_{\mathrm{S}}^{0} \mathrm{~K}_{\mathrm{S}}^{0} \pi^{+} \pi^{-}, \mathrm{K}_{\mathrm{S}}^{0} \mathrm{~K}_{\mathrm{L}}^{0} \pi^{+} \pi^{-}$, 


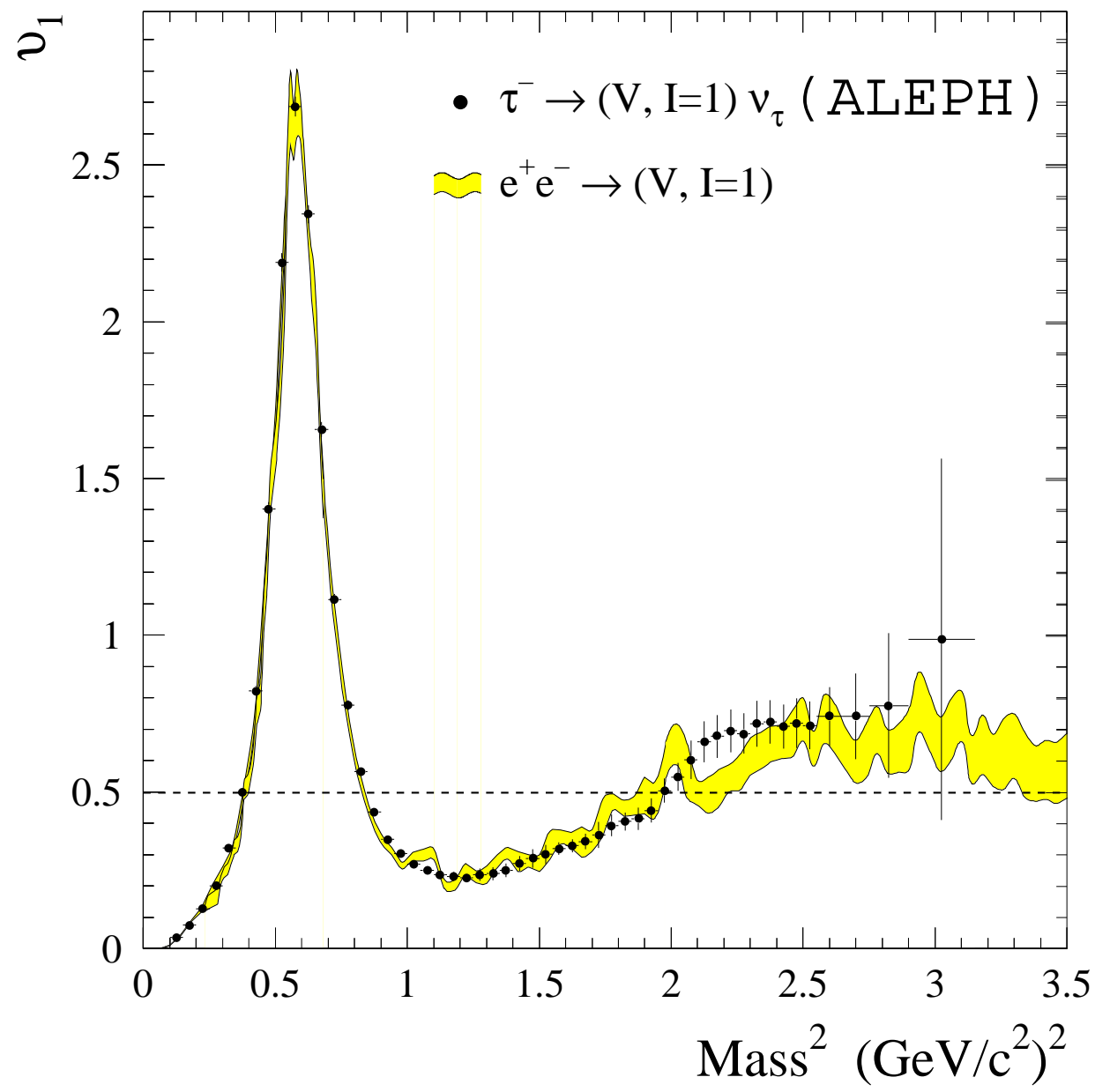

Figure 12: Total hadronic vector current spectral function from $\tau$ decays (data points) and the corresponding distribution calculated from $e^{+} e^{-}$isovector states using isospin symmetry. The shaded band includes statistical and systematic errors. The dashed line corresponds to the naive isovector quark-parton prediction.

$\mathrm{K}_{\mathrm{S}}^{0} \mathrm{~K}^{+} \pi^{-} \pi^{0}$ and $\mathrm{K}_{\mathrm{S}}^{0} \mathrm{~K}^{-} \pi^{+} \pi^{0}$. With the assumption that the cross sections for the processes $e^{+} e^{-} \rightarrow \mathrm{K}^{0} \overline{\mathrm{K}}^{0}(\pi \pi)^{0}$ and $e^{+} e^{-} \rightarrow \mathrm{K}^{+} \mathrm{K}^{-}(\pi \pi)^{0}$ are equal, one can summarize the total $\mathrm{KK} \pi \pi$ contribution as twice the above corrected $\mathrm{K}_{\mathrm{S}}^{0}+X$ cross section. A reasonable estimate of the systematic uncertainty, implied by the assumption made, is obtained by taking the cross section for the channel $\mathrm{K}^{+} \mathrm{K}^{-} \pi^{+} \pi^{-}$measured by DM1 [72] and DM2 [61]. Since the $\mathrm{KK} \pi \pi$ isovector part is unknown it is assumed to be $(50 \pm 50) \%$.

Fig. 12 shows both the total $\tau$ vector current spectral function and the corresponding spectral function coming from the isovector $e^{+} e^{-}$cross section. Agreement is found at low mass-squared. Above $2 \mathrm{GeV}^{2} / c^{4}$, the $\tau$ data are somewhat higher than the $e^{+} e^{-}$ measurements (note that the $\tau$ data points are highly correlated - see Fig. 5). This is essentially due to the observed disagreement between the $\tau^{-} \rightarrow 2 \pi^{-} \pi^{+} \pi^{0} \nu_{\tau}$ spectral function and the corresponding $e^{+} e^{-}$cross section. 


\subsection{A Fit of the Pion Form Factor}

Several parametrizations of the pion form factor Eq. (5) can be found in the literature. (See, e.g., Refs. [2, 3, 44, 73].) In this section, different fits using the Kühn-Santamaria [2] and the Gounaris-Sakurai parametrizations [3] are presented. In addition, a combined fit to $\tau$ and $e^{+} e^{-}$data is performed, where the masses and widths of the $\rho^{ \pm}(770)$ and the $\rho^{0}(770)$ are separately determined, in order to extract possible isospin violating effects.

As seen in Section 6.3, the unfolding procedure introduces additional systematic uncertainties because of the numerical instability of the problem. Generally, one can state that unfolding is necessary if a theoretical description of an observed distribution is not available, as is the case for the total vector (and axial-vector) hadronic spectral functions in $\tau$ decays. Also, unfolding is needed for comparison with $e^{+} e^{-}$results where the mass is experimentally known with very good accuracy. However in the specific case of the $\pi^{-} \pi^{0}$ spectral function, phenomenological models based on vector resonances which describe the lineshape exist. One therefore does not need to unfold, as a convolution of the theoretical curve with the detector response matrix $A$ is a well defined and stable problem. The convolved theoretical distribution can subsequently be fitted to the data. This procedure is followed here.

The results of all types of fits are given with errors, including both statistical and systematic uncertainties. The sources of systematic uncertainties correspond in detail to those mentioned in Section 6, apart from those introduced by the unfolding procedure. The correlations between the fitted parameters in the combined fit are given as a correlation matrix.

Systematic errors of the $e^{+} e^{-}$annihilation data are caused by uncertainties coming mainly from the determination of the efficiency of the two-pion reconstruction and the luminosity measurement. These errors are given as normalization uncertainties by the experiments, i.e., they scale linearly with the measured cross sections. The usual way of introducing such errors into a least square minimization is to treat them as being totally correlated. They therefore populate the off-diagonal elements of the corresponding covariance matrix. However, it is known that this procedure introduces a bias into the minimization, leading systematically to lower values in terms of the normalization of the fitted parametrization [74]. To avoid such an effect, the best estimate of the parameters is found when using systematic errors as the statistical ones without correlations. The corresponding parameter errors, however, are determined by repeating the fit when taking into account the full correlations of the systematic errors among the measurements of one experiment. Measurements between different experiments are assumed to be uncorrelated.

\subsubsection{The Kühn-Santamaria (KS) Parametrization}

In the Kühn-Santamaria parametrization the pion form factor is given by contributions from the known isovector meson resonances $\rho(770), \rho(1450)$ and $\rho(1700)$, taking into account $\rho-\omega$ interference:

$$
F_{\pi}^{I=1,0}(s)=\frac{\mathrm{BW}_{\rho(770)}(s) \frac{1+\delta \mathrm{BW}_{\omega(783)}(s)}{1+\delta}+\beta \mathrm{BW}_{\rho(1450)}(s)+\gamma \mathrm{BW}_{\rho(1700)}(s)}{1+\beta+\gamma}
$$


with the Breit-Wigner propagators

$$
\mathrm{BW}_{\rho\left(M_{\rho}\right)}^{\mathrm{KS}}(s)=\frac{M_{\rho}^{2}}{M_{\rho}^{2}-s-i \sqrt{s} \Gamma_{\rho}(s)}
$$

and the energy dependent width

$$
\Gamma_{\rho}(s)=\Gamma_{\rho}\left(M_{\rho}^{2}\right)\left(\frac{M_{\rho}^{2}}{s}\right)^{\lambda}\left(\frac{k(s)}{k\left(M_{\rho}^{2}\right)}\right)^{3},
$$

where $k(s)=\frac{1}{2} \sqrt{s} \beta_{\pi}(s)$ and $k\left(M_{\rho}^{2}\right)$ is the pion momentum in the $\rho$ rest frame. As in Refs. [2] and [44] the amplitudes $\beta, \gamma$ and $\delta$ are assumed to be real. Interference with the isospin-violating electromagnetic $\omega \rightarrow \pi^{+} \pi^{-}$decay occurs only in $e^{+} e^{-}$annihilation. Consequently, $\delta$ is fixed to zero when fitting $\tau$ data. According to Ref. [73] a fit parameter $\lambda$ is added to take into account possible uncertainties in the $p$-wave approximation of the $s$-dependent width.

\begin{tabular}{|c||c|c|}
\hline Parameter & Kühn-Santamaria & Gounaris-Sakurai \\
\hline \hline$M_{\rho^{ \pm}(770)}$ & $774.9 \pm 0.9$ & $776.4 \pm 0.9$ \\
$\Gamma_{\rho^{ \pm}(770)}$ & $144.2 \pm 1.5$ & $150.5 \pm 1.6$ \\
\hline$\beta$ & $-0.094 \pm 0.007$ & $-0.077 \pm 0.008$ \\
$M_{\rho^{ \pm}(1450)}$ & $1363 \pm 15$ & $1400 \pm 16$ \\
$\Gamma_{\rho(1450)}$ & $\equiv 310$ & $\equiv 310$ \\
\hline$\gamma$ & $-0.015 \pm 0.008$ & $0.001 \pm 0.009$ \\
$M_{\rho^{ \pm}(1700)}$ & $\equiv 1700$ & $\equiv 1700$ \\
$\Gamma_{\rho(1700)}$ & $\equiv 235$ & $\equiv 235$ \\
\hline$\lambda$ & $\equiv 1.0$ & $\equiv 1.0$ \\
\hline \hline$\chi^{2} /$ dof & $81 / 65$ & $54 / 65$ \\
\hline
\end{tabular}

Table 2: Fit results of the pion form factor in $\tau^{-} \rightarrow \pi^{-} \pi^{0} \nu_{\tau}$ decays using the KühnSantamaria (left-hand column) and the Gounaris-Sakurai parametrization (right-hand column). The values of $\Gamma_{\rho(1450)}, M_{\rho^{ \pm}(1700)}$ and $\Gamma_{\rho(1700)}$ are taken from Ref. [8].

The results of the $\tau$ data and the combined fit using the KS parametrization are listed in the left-hand columns of Tables 2 and 3.

\subsubsection{The Gounaris-Sakurai (GS) Parametrization}

Starting from a more elaborate treatment of the $p$-wave scattering amplitude for a broad resonance, the following parametrization was obtained with the additional requirement of the normalization $F_{\pi}(0) \equiv 1$, as in the KS parametrization. The simple Breit-Wigner resonances in (14) are replaced (for $s \geq 4 m_{\pi}^{2}$ ) by [3]

$$
\mathrm{BW}_{\rho\left(M_{\rho}\right)}^{\mathrm{GS}}(s)=\frac{M_{\rho}^{2}\left(1+d \cdot \Gamma_{\rho} / M_{\rho}\right)}{M_{\rho}^{2}-s+f(s)-i \sqrt{s} \Gamma_{\rho}(s)},
$$


where

$$
f(s)=\Gamma_{\rho} \frac{M_{\rho}^{2}}{k^{3}\left(M_{\rho}^{2}\right)}\left[k^{2}(s)\left(h(s)-h\left(M_{\rho}^{2}\right)\right)+\left.\left(M_{\rho}^{2}-s\right) k^{2}\left(M_{\rho}^{2}\right) \frac{d h}{d s}\right|_{s=M_{\rho}^{2}}\right] .
$$

The $s$ dependence of $\Gamma_{\rho}(s)$ and $k(s)$ is the same as in Eq. (16). The function $h(s)$ is defined as

$$
h(s)=\frac{2}{\pi} \frac{k(s)}{\sqrt{s}} \ln \frac{\sqrt{s}+2 k(s)}{2 m_{\pi}},
$$

with $d h /\left.d s\right|_{M_{\rho}^{2}}=h\left(M_{\rho}^{2}\right)\left[\left(8 k^{2}\left(M_{\rho}^{2}\right)\right)^{-1}-\left(2 M_{\rho}^{2}\right)^{-1}\right]+\left(2 \pi M_{\rho}^{2}\right)^{-1}$.

The normalization $\mathrm{BW}_{\rho\left(M_{\rho}\right)}^{\mathrm{GS}}(0)=1$ fixes the parameter $d=f(0) /\left(\Gamma_{\rho} M_{\rho}\right)$. It is found to be $[3]$

$$
d=\frac{3}{\pi} \frac{m_{\pi}^{2}}{k^{2}\left(M_{\rho}^{2}\right)} \ln \frac{M_{\rho}+2 k\left(M_{\rho}^{2}\right)}{2 m_{\pi}}+\frac{M_{\rho}}{2 \pi k\left(M_{\rho}^{2}\right)}-\frac{m_{\pi}^{2} M_{\rho}}{\pi k^{3}\left(M_{\rho}^{2}\right)} .
$$

The results of the $\tau$ data and the combined fit using the GS parametrization are listed in the right-hand columns of the Tables 2 and 3.

Concluding from Table 2, the fits establish a need for the $\rho(1450)$ contribution to the weak pion form factor in the KS and GS parametrizations $(\beta=-0.087 \pm$ $0.012)$ with a fitted mass $M_{\rho(1450)}=(1380 \pm 24) \mathrm{MeV} / c^{2}$ when fixing the width at $\Gamma_{\rho(1450)}=310 \mathrm{MeV} / c^{2}$ [8]. No significant evidence of a $\rho(1700)$ contribution is found $(\gamma=-0.008 \pm 0.008)$. The previous values are the weighted averages between the results of both fit types. Their errors account for statistical and systematic uncertainties coming from model dependence. It must be stated that the fitted $\rho(1450)$ parameters show large correlations with the corresponding $\rho(1450)$ width. In fact, fixing $\Gamma_{\rho(1450)}=600 \mathrm{MeV} / \mathrm{c}^{2}$ leads to the averaged fit results: $\beta=-0.156, M_{\rho(1450)}=1470 \mathrm{MeV} / c^{2}$ and $\gamma=-0.030$ with a substantial improvement of the $\chi^{2}$, i.e., 56 (KS) and 51 (GS) over 65 degrees of freedom.

One could try to explain the enhancement of the pion form factor centered around $1200 \mathrm{MeV} / \mathrm{c}^{2}$ as originating from an inelastic effect induced via unitarity by the opening of the $\omega \pi^{0}$ channel which occurs at $920 \mathrm{MeV} / c^{2}$ [75]. Although this effect is physically sound and should take place, the proposed description is not very predictive and requires a factor $\left(M_{0}^{2} /\left(M_{0}^{2}-s-i M_{0} \Gamma_{0}\right)\right)^{n_{0}}$ with three additional parameters $M_{0}, \Gamma_{0}$ and $n_{0}$ to be adjusted in the fit. However, the existence of a $\rho(1450)$ meson is already well established in the $\pi^{+} \pi^{-} 2 \pi^{0}$ final state [76] and since the sensitivity of the data on the pion form factor is not sufficient to fit a larger number of parameters, the inelastic parametrization is not used in the present analysis.

Fig. 13 shows the KS/GS-type fits using one and three Breit-Wigner amplitudes.

\subsubsection{Combined Fit of $\tau$ and $e^{+} e^{-}$Data}

The results of the combined $\tau$ and $e^{+} e^{-}$data fit with the KS and GS parametrizations are presented in Table 3. In these fits, the pion form factor is described by the $\rho$ resonance with different parameters fitted for $\rho^{ \pm}$and $\rho^{0}$, while the much smaller $\rho(1450)$ and 


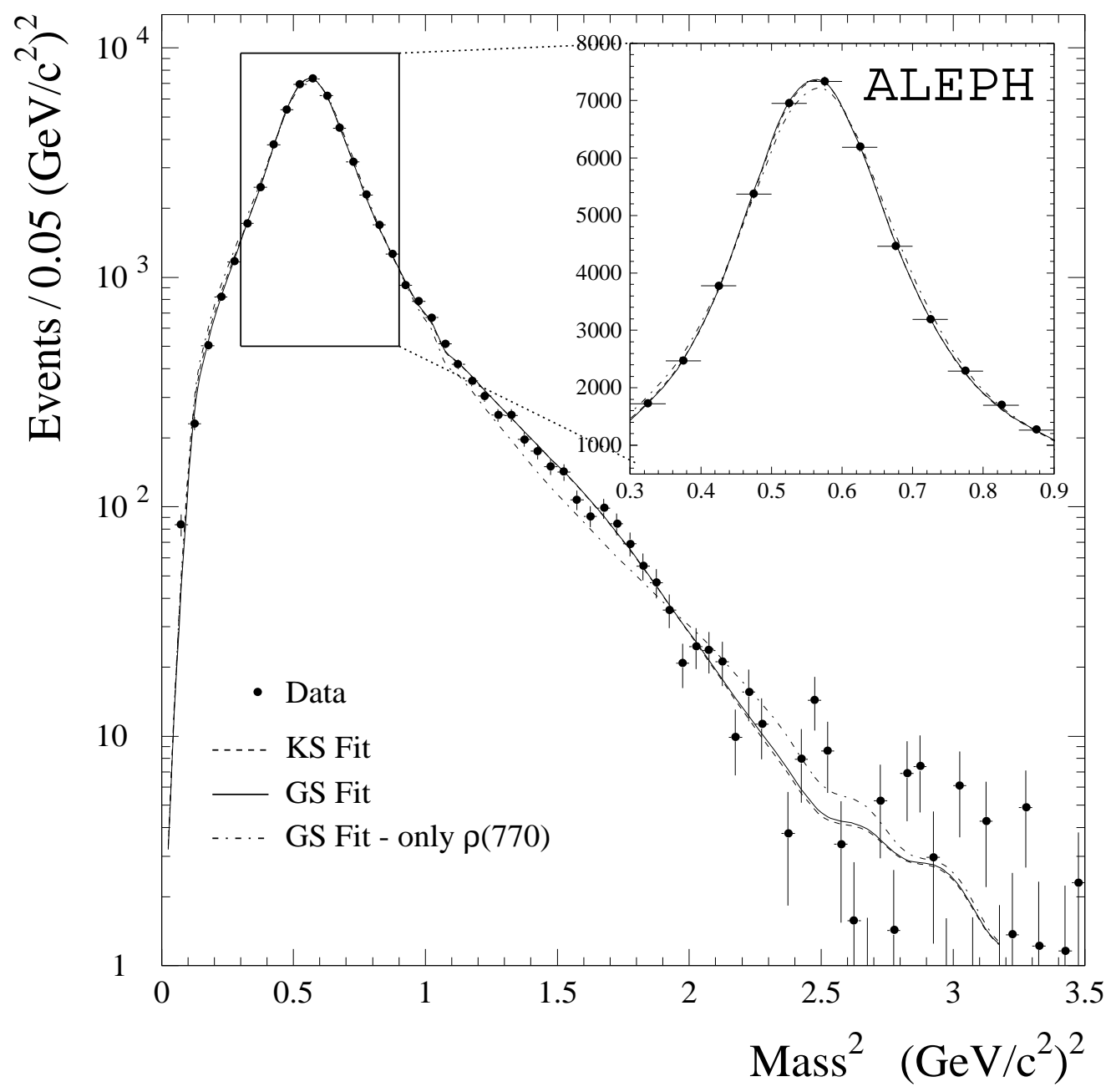

Figure 13: Fit of the $\tau^{-} \rightarrow \pi^{-} \pi^{0} \nu_{\tau}$ invariant mass spectrum using the Kühn-Santamaria $(K S)$ and the Gounaris-Sakurai (GS) parametrization. The solid and dashed curves are the functions corresponding to the KS/GS-type form factor fits given in Table 2. They have been convolved with the detector resolution and the $\tau$ phase space. Due to statistical fluctuations in the detector response matrix, the functions are not smooth after convolution. The dashed-dotted line corresponds to a GS-type fit in which only the $\rho(770)$ contribution is taken into account. 
$\rho(1700)$ contributions are assumed to be isospin invariant. In this way, it is possible to directly compare, for the first time, in a model-independent way the parameters of the charged and the neutral $\rho$ 's. Due to the large number of degrees of freedom in the combined fits, all free parameters can be simultaneously determined with good precision. All presented fits resulted in significantly higher $\rho(770)$ masses than the value of $M_{\rho(770)}=(768.5 \pm 0.6) \mathrm{MeV} / c^{2}$ (average of $\rho^{ \pm}$and $\rho^{0}$ ) given by the PDG [8]. Within

\begin{tabular}{|c||c||c|c|}
\hline Parameter & Kühn-Santamaria $(\mathrm{KS})$ & \multicolumn{2}{c|}{ Gounaris-Sakurai $(\mathrm{GS})$} \\
\hline \hline$\delta$ & $(1.91 \pm 0.15) \times 10^{-3}$ & $(1.97 \pm 0.10) \times 10^{-3}$ & $(1.97 \pm 0.15) \times 10^{-3}$ \\
\hline$M_{\rho^{ \pm}(770)}$ & $773.4 \pm 0.9$ & $775.7 \pm 0.9$ & $783.8 \pm 3.0$ \\
$M_{\rho^{0}(770)}$ & $773.4 \pm 0.7$ & $775.7 \pm 0.7$ & $783.8 \pm 3.0$ \\
\hline$\Gamma_{\rho^{ \pm}(770)}$ & $147.7 \pm 1.6$ & $150.8 \pm 1.7$ & $162.0 \pm 5.3$ \\
$\Gamma_{\rho^{0}(770)}$ & $147.3 \pm 1.3$ & $150.8 \pm 1.3$ & $162.4 \pm 5.0$ \\
\hline$\beta$ & $-0.229 \pm 0.020$ & $-0.161 \pm 0.010$ & $-0.184 \pm 0.010$ \\
$M_{\rho(1450)}$ & $1465 \pm 22$ & $1448 \pm 19$ & $1490 \pm 23$ \\
$\Gamma_{\rho(1450)}$ & $696 \pm 47$ & $503 \pm 38$ & $591 \pm 53$ \\
\hline$\gamma$ & $0.075 \pm 0.022$ & $0.076 \pm 0.009$ & $0.074 \pm 0.010$ \\
$M_{\rho(1700)}$ & $1760 \pm 31$ & $1757 \pm 20$ & $1799 \pm 34$ \\
$\Gamma_{\rho(1700)}$ & $215 \pm 86$ & $237 \pm 78$ & $255 \pm 39$ \\
\hline$\lambda$ & $\equiv 1.0$ & $\equiv 1.0$ & $0.45 \pm 0.11$ \\
\hline \hline \multicolumn{2}{|c||}{$190 / 195$} & $194 / 195$ & $193 / 194$ \\
\hline$\chi^{2} /$ dof & $0.0 \pm 1.0$ & $0.0 \pm 1.0$ & $0.0 \pm 1.2$ \\
\hline$M_{\rho^{ \pm}(770)}-M_{\rho^{0}(770)}$ & $0.4 \pm 1.8$ & $0.0 \pm 2.0$ & $-0.4 \pm 2.5$ \\
$\Gamma_{\rho^{ \pm}(770)}-\Gamma_{\rho^{0}(770)}$ & \multicolumn{3}{|c|}{} \\
\hline
\end{tabular}

Table 3: Results of the combined fit of the $\pi^{ \pm} \pi^{0}$ and $\pi^{+} \pi^{-}$resonance amplitudes according to the Kühn-Santamaria and the Gounaris-Sakurai model. In the second GS-type fit, the parameter $\lambda$ introduced in Eq. (16) is additionally fitted. This leads to higher parameter errors with strong correlations among them.

\begin{tabular}{|c||c|c|c|c|}
\hline ALEPH & $M_{\rho}^{ \pm}$ & $M_{\rho}^{0}$ & $\Gamma_{\rho}^{ \pm}$ & $\Gamma_{\rho}^{0}$ \\
\hline \hline$M_{\rho}^{ \pm}$ & 1 & 0.18 & 0.32 & 0.02 \\
\hline$M_{\rho}^{0}$ & - & 1 & 0.03 & 0.31 \\
\hline$\Gamma_{\rho}^{ \pm}$ & - & - & 1 & 0.17 \\
\hline
\end{tabular}

Table 4: Average correlations found in the KS/GS-type fits (with fixed $\lambda$ ) between masses and widths of the charged and neutral $\rho^{0}(770)$.

large uncertainties (about $8 \mathrm{MeV} / \mathrm{c}^{2}$ ) essentially due to model dependence, the width $\Gamma_{\rho(770)}$ was found to be in agreement with the PDG value of $\Gamma_{\rho(770)}=(150.7 \pm 1.2) \mathrm{MeV} / \mathrm{c}^{2}$. The additional fit parameter $\lambda$ in the second type of fit is found to be $\lambda=0.45 \pm 0.11$, 
i.e., quite different from the fixed value $\lambda \equiv 1$ in the first type of fits. As can be expected, a different adjustment of $\lambda$ has a considerable impact on the fitted mass and width of the $\rho(770)$. Both $\tau$ and $e^{+} e^{-}$data are sensitive to the $\rho(1450)$ parameters. The $\rho(1450)$ width is found to be strongly model-dependent, but from all fit types its value is significantly higher than the PDG value of $\Gamma_{\rho(1450)}=(310 \pm 60) \mathrm{MeV} / \mathrm{c}^{2}$ obtained from $e^{+} e^{-} \rightarrow \omega \pi$ data. This difference could be linked to the neglect of inelastic effects as discussed in the previous section. The fitted masses $M_{\rho(1450)}$ from all fit types are found to be in rather good agreement with the PDG average of $M_{\rho(1450)}=(1449 \pm 8) \mathrm{MeV} / \mathrm{c}^{2}$. The information concerning the mass, width and relative amplitude of the $\rho(1700)$ is essentially extracted from the $e^{+} e^{-}$data and found to be in fairly good agreement with the PDG values.

Systematic uncertainties due to the energy scale in $e^{+} e^{-}$annihilation experiments are difficult to estimate as, in general, the publications do not refer to this point. In most cases, the experiments used the narrow $\phi(1020)$ resonance peak to calibrate the beam energy. Consequently, intrinsic uncertainties are introduced by slight modifications of the central $\phi$ mass value over the years, e.g., $M_{\phi}=1019.57 \mathrm{MeV} / c^{2}$ in 1980 became $M_{\phi}=1019.41 \mathrm{MeV} / c^{2}$ in 1996. An additional systematic uncertainty of $0.3 \mathrm{MeV} / c^{2}$ is considered in the $\rho^{0}$ mass measurement.

Although the absolute values of the $\rho(770)$ masses and widths depend significantly on the KS- or GS-type of the fit and the parameter $\lambda$, their respective differences, i.e., $\Delta M_{\rho(770)}=M_{\rho^{ \pm}(770)}-M_{\rho^{0}(770)}$ and $\Delta \Gamma_{\rho(770)}=\Gamma_{\rho^{ \pm}(770)}-\Gamma_{\rho^{0}(770)}$ are stable. Using the fit results from Table 3, one obtains the average

$$
\begin{aligned}
\Delta M_{\rho(770)} & =(0.0 \pm 1.0 \pm 0.1) \mathrm{MeV} / c^{2} \\
\Delta \Gamma_{\rho(770)} & =(0.1 \pm 1.8 \pm 0.5) \mathrm{MeV} / c^{2}
\end{aligned}
$$

The first errors are due to statistical and systematic uncertainties (including correlations between the fit parameters), while the second ones account for differences from the resonance parametrizations. Fig. 14 shows the results with its $39 \%$ CL error ellipse taking into account the correlations between the fit parameters given in Table 4.

A difference between $\Gamma_{\rho^{ \pm}}$and $\Gamma_{\rho^{0}}$ could occur on one hand through electromagnetic isospin-violating decay modes such as $\rho \rightarrow \pi \pi \gamma$, which is observed at the $1 \%$ level for the $\rho^{0}$ [8]. On the other hand the dominant $\rho \rightarrow \pi \pi$ channel could also manifest some isospin violation. An obvious contribution comes from the observed $\pi^{ \pm}-\pi^{0}$ and a potential $\rho^{ \pm}$$\rho^{0}$ mass differences which reflect into different values for the width according to (16). The $\rho$ mass dependence is not completely clear: one could consider a variation given by

$$
\Gamma_{\rho} \sim k^{3}\left(M_{\rho}^{2}\right) / M_{\rho}^{2}
$$

or, as argued in Chiral Perturbation Theory [77],

$$
\Gamma_{\rho} \sim k^{3}\left(M_{\rho}^{2}\right)
$$

According to the charge of the $\rho$, the pion momentum in the $\rho$ rest system is given by $2 k\left(M_{\rho^{0}}^{2}\right)=\left(M_{\rho^{0}}^{2}-4 m_{\pi^{ \pm}}^{2}\right)^{1 / 2}$ for the neutral $\rho^{0}$ and $2 k\left(M_{\rho^{ \pm}}^{2}\right)=\left[M_{\rho^{ \pm}}^{2}-2\left(m_{\pi^{ \pm}}^{2}+\right.\right.$ 


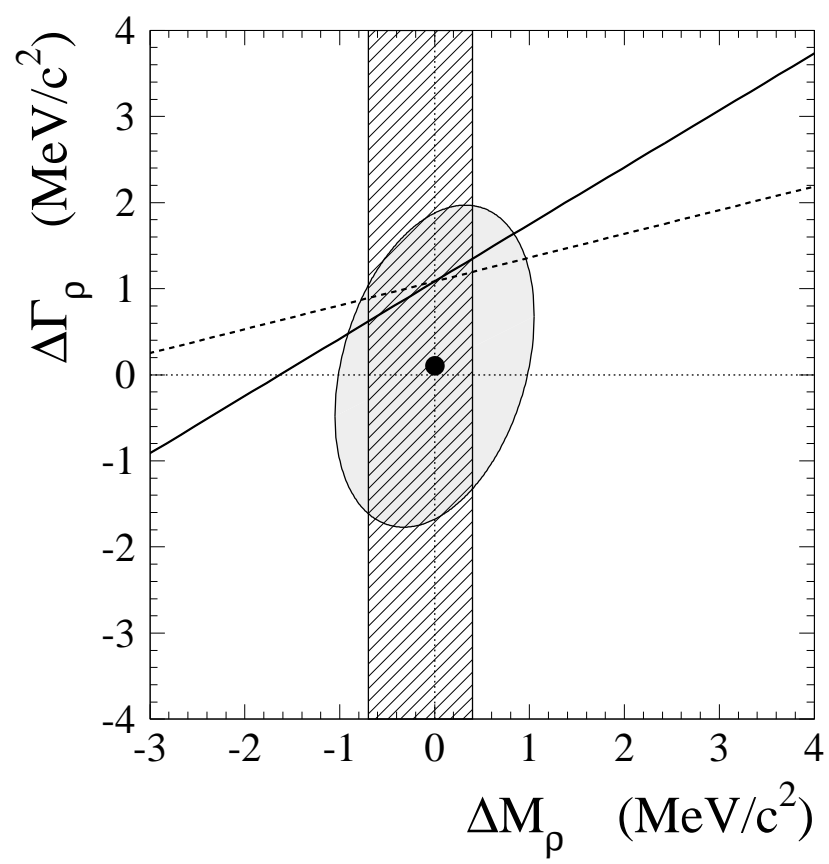

Figure 14: Width difference $\Delta \Gamma_{\rho(770)}=\Gamma_{\rho^{ \pm}(770)}-\Gamma_{\rho^{0}(770)}$ as a function of the difference in the $\rho(770)$ mass $\Delta M_{\rho(770)}=M_{\rho^{ \pm}(770)}-M_{\rho^{0}(770)}$. The point is the measurement with its correlated one-sigma error ellipse. The dashed and solid lines show the expected dependences from Eqs. (21) and (22), respectively. The hatched area depicts the electromagnetic $\rho$ mass difference predicted in [78].

$\left.\left.m_{\pi^{0}}^{2}\right)+\left(m_{\pi^{ \pm}}^{2}-m_{\pi^{0}}^{2}\right)^{2} / M_{\rho^{ \pm}}^{2}\right]^{1 / 2}$ for the charged $\rho^{ \pm}$, respectively. The dashed and solid lines in Fig. 14 give the functional dependence of the width difference $\Delta \Gamma_{\rho}$ on $\Delta M_{\rho}$ in the approximations of Eqs. (21) and (22), respectively, normalized to the fitted value of $\Gamma_{\rho}$.

It is interesting to observe that the measured $\Delta M_{\rho}$ is significantly smaller than the mass difference between charged and neutral pions $\Delta M_{\pi}=M_{\pi^{ \pm}}-M_{\pi^{0}}=(4.5936 \pm$ $0.0007) \mathrm{MeV} / \mathrm{c}^{2}$ [8], where the dominant effect is understood to be of electromagnetic origin $\left(\Delta M_{\pi}^{\mathrm{em}} \simeq 4.5 \mathrm{MeV} / c^{2}[79]\right)$. The $\Delta M_{\rho}$ measurement can be compared to the (model dependent) result of $\Delta M_{\rho}=(-0.3 \pm 2.2) \mathrm{MeV} / c^{2}$ [8] obtained in hadronic production, however in good agreement with this determination. The Mark III Collaboration exploited data on $J / \psi \rightarrow \pi^{+} \pi^{-} \pi^{0}$ decays, dominated by $J / \psi \rightarrow \rho \pi$, to measure the mass difference of the charged and neutral $\rho$ 's [80]. Their preliminary result is found to be in good agreement with the result presented here. Note that the value deduced from the difference in the mean values taken from Ref. [8] $\left\langle M_{\rho^{ \pm}}\right\rangle-\left\langle M_{\rho^{0}}\right\rangle=$ $(-1.8 \pm 1.4) \mathrm{MeV} / c^{2}$ is potentially unreliable as they both represent the weighted mean of independent measurements using not necessarily the same parametrizations. A theoretical electromagnetic $\rho$ mass difference of $-0.7 \mathrm{MeV} / c^{2}<\Delta M_{\rho}<0.4 \mathrm{MeV} / c^{2}$ in agreement with the measurement has recently been evaluated in Ref. [78]. The measured difference 
$\Delta \Gamma_{\rho}$ is found to be consistent with the expected isospin violation from the $\pi^{ \pm}-\pi^{0}$ and $\rho^{ \pm}-\rho^{0}$ mass differences.

\section{Conclusions}

Measurements are presented of the non-strange $\tau$ vector current spectral functions, with special emphasis on the two- and four-pion final states. Their distributions and the corresponding error matrices can be obtained as postscript and data files from the ALEPH publication server on the $\mathrm{WWW}^{4}$.

The CVC property of the Standard Model provides the possibility to identify the $\tau$ vector decay channels with the isovector components of the $e^{+} e^{-}$hadronic final states by means of isospin symmetry. The $\pi^{+} \pi^{-}$and $\pi^{+} \pi^{-} \pi^{+} \pi^{-}$cross sections measured in $e^{+} e^{-}$ annihilation have been found to be in good agreement with the respective $\pi^{-} \pi^{0}$ and $\pi^{-} 3 \pi^{0}$ spectral functions. The $\pi^{+} \pi^{-} \pi^{0} \pi^{0}$ cross section has been compared to the corresponding linear combination of $\pi^{-} 3 \pi^{0}$ and $2 \pi^{-} \pi^{+} \pi^{0}$ spectral functions from $\tau$ decays. Above $2 \mathrm{GeV}^{2} / c^{4}, \tau$ data points are significantly higher than the respective $e^{+} e^{-}$measurements (DM2). This is thought to be due to a disagreement in the non-resonant contribution as a good agreement with DM2 for the resonant $(\omega \pi)$ part of the cross section has recently been found by the ALEPH Collaboration [6].

Fits of the pion form factor based on the Kühn-Santamaria [2] and the GounarisSakurai [3] parametrizations have been performed. In this framework, the existence of an additional $\rho(1450)$ contribution is firmly established in $\tau$ decays. The fit using the GS parametrization resulted in a better description of $\tau$ data yielding a $\chi^{2}$ of 54 over 65 degrees of freedom. The parameters of the dominant $\rho(770)$ contribution found in this fit are: $M_{\rho^{ \pm}(770)}=(776.4 \pm 0.9) \mathrm{MeV} / c^{2}$ and $\Gamma_{\rho^{ \pm}(770)}=(150.5 \pm 1.6) \mathrm{MeV} / c^{2}$.

A combined fit to $\tau$ and $e^{+} e^{-}$data has been performed in order to measure the difference in mass and width between the dominant charged and neutral $\rho(770)$ amplitudes, expected to be generated by isospin-violating effects. The observed mass difference $M_{\rho^{ \pm}(770)}-M_{\rho^{0}(770)}=(0.0 \pm 1.0) \mathrm{MeV} / c^{2}$ is significantly smaller than the corresponding value for the pions, while the width difference $\Gamma_{\rho^{ \pm}(770)}-\Gamma_{\rho^{0}(770)}=$ $(0.1 \pm 1.9) \mathrm{MeV} / c^{2}$ is consistent with isospin violation from the $\pi^{ \pm}-\pi^{0}$ and $\rho^{ \pm}-\rho^{0}$ mass differences.

\section{Acknowledgments}

We gratefully acknowledge our colleagues from the accelerator divisions for the successful operation of LEP. We are indebted to the engineers and technicians in all our institutions for their contribution to the excellent performance of ALEPH. Those of us from nonmember countries thank CERN for its hospitality.

\footnotetext{
${ }^{4}$ http://alephwww.cern.ch/ALPUB/paper/paper.html
} 


\section{Appendix}

In the classification developed by Pais [36], pion isospin states are organized in symmetry classes with orthogonal wave functions. To each isospin class $\{i j k\}$ corresponds a partial width $\Gamma_{i j k}$ in $\tau$ decays and a cross section $\sigma_{i j k}$ in $e^{+} e^{-}$annihilation. In these terms, the four-pion isovector states are linear combinations of the classes $\{310\}$ and $\{211\}$ :

$$
\begin{aligned}
\Gamma_{\pi^{ \pm} 3 \pi^{0}} & =\frac{2}{5} \Gamma_{310}, \\
\Gamma_{3 \pi^{ \pm} \pi^{0}} & =\Gamma_{211}+\frac{3}{5} \Gamma_{310}, \\
\sigma_{\pi^{+} \pi^{-} \pi^{+} \pi^{-}} & =\frac{4}{5} \sigma_{310}, \\
\sigma_{\pi^{+} \pi^{-} \pi^{0} \pi^{0}} & =\sigma_{211}+\frac{1}{5} \sigma_{310} .
\end{aligned}
$$

For the six-pion $\tau$ final states one obtains the following decompositions [33, 81]:

$$
\begin{aligned}
\Gamma_{\pi^{-} 5 \pi^{0}} & =\frac{9}{35} \Gamma_{510} \\
\Gamma_{2 \pi^{-} \pi^{+} 3 \pi^{0}} & =\frac{2}{7} \Gamma_{510}+\frac{1}{5} \Gamma_{411}+\frac{4}{5} \Gamma_{330}+\frac{1}{2} \Gamma_{321}, \\
\Gamma_{3 \pi^{-} 2 \pi^{+} \pi^{0}} & =\frac{16}{35} \Gamma_{510}+\frac{4}{5} \Gamma_{411}+\frac{1}{5} \Gamma_{330}+\frac{1}{2} \Gamma_{321}
\end{aligned}
$$

and for the $e^{+} e^{-}$states:

$$
\begin{aligned}
\sigma_{\pi^{-} \pi^{+} 4 \pi^{0}} & =\frac{3}{35} \sigma_{510}+\frac{3}{5} \sigma_{411} \\
\sigma_{2 \pi^{-} 2 \pi^{+} 2 \pi^{0}} & =\frac{8}{35} \sigma_{510}+\frac{2}{5} \sigma_{411}+\frac{2}{5} \sigma_{330}+\sigma_{321} \\
\sigma_{3 \pi^{-} 3 \pi^{+}} & =\frac{24}{35} \sigma_{510}+\frac{3}{5} \sigma_{330} .
\end{aligned}
$$

\section{References}

[1] Y.S. Tsai, Phys. Rev. D4 (1971) 2821.

[2] J.H. Kühn and A. Santamaria, Z. Phys. C48 (1990) 445.

[3] G.J. Gounaris and J.J. Sakurai, Phys. Rev. Lett. 21 (1968) 244.

[4] H. Albrecht et al. (ARGUS Collaboration), Phys. Lett. B185 (1987) 223.

[5] W.T. Ford, Nucl. Phys. B40 (Proc. Suppl.) (1995) 191.

[6] D. Buskulic et al. (ALEPH Collaboration), CERN PPE/96-103.

[7] A. Höcker (for the ALEPH Collaboration), 'Vector and Axial-Vector Spectral Functions and QCD', Talk given at the TAU'96 Conference, Colorado (1996). 
[8] R.M. Barnett et al. (Particle Data Group), Phys. Rev. D54 (1996) 1.

[9] W.J. Marciano and A. Sirlin, Phys. Rev. Lett. 56 (1986) 22.

[10] J.Z. Bai et al. (BES Collaboration), Phys. Rev. D53 (1996) 20.

[11] E. Braaten, S. Narison and A. Pich, Nucl. Phys. B373 (1992) 581.

[12] A. Pich, Nucl. Phys. B39 (Proc. Suppl.) (1995) 326.

[13] D. Decamp et al. (ALEPH Collaboration), Nucl. Inst. Meth. A294 (1990) 121.

[14] D. Buskulic et al. (ALEPH Collaboration), Nucl. Inst. Meth. A360 (1995) 481.

[15] S. Jadach, J.H. Kühn and Z. Wa̧s, Comp. Phys. Comm. 64 (1991) 275.

[16] S. Jadach, B.F.L. Ward and Z. Wạs, Comp. Phys. Comm. 79 (1994) 503.

[17] S. Jadach et al., Comp. Phys. Comm. 76 (1993) 361.

[18] R. Brun et al., GEANT3.15, CERN DD/EE/84-1.

[19] D. Buskulic et al. (ALEPH Collaboration), Z. Phys. C62 (1994) 539.

[20] D. Buskulic et al. (ALEPH Collaboration), Z. Phys. C70 (1996) 561.

[21] D. Buskulic et al. (ALEPH Collaboration), Z. Phys. C70 (1996) 579.

[22] D. Decamp et al. (ALEPH Collaboration), Z. Phys. C54 (1992) 211.

[23] A. Höcker and V. Kartvelishvili, Nucl. Inst. Meth. A372 (1996) 469.

[24] D. Gibaut et al. (CLEO Collaboration), Phys. Rev. Lett. 73 (1994) 934.

[25] R. Balest et al. (CLEO Collaboration), Phys. Rev. Lett 75 (1995) 3809.

[26] M. Artuso et al. (CLEO Collaboration), Phys. Rev. Lett. 69 (1992) 3278.

[27] T.E. Coan et al. (CLEO-Collaboration), Phys. Rev. D53 (1996) 6037.

[28] S. Towers (for the OPAL Collaboration), 'Tau decays involving neutral kaons'; R. Kass (for the CLEO Collaboration), 'Three-prong tau decays with kaons'; Talks given at the TAU'96 Conference, Colorado (1996).

[29] P.L. Campana (for the ALEPH Collaboration), 'Branching fractions for modes involving kaons', Talk given at the TAU'96 Conference, Colorado (1996).

[30] D. Buskulic et al. (ALEPH Collaboration), Phys. Lett. B332 (1994) 209.

[31] H.J. Park, Ph.D. Thesis, Université de Paris-Sud, Report LAL-95-08 (1995) 245.

[32] M. Davier, ' $\tau$ decays into strange particles and QCD', Talk given at the TAU'96 Conference, Colorado (1996). 
[33] A. Rougé, Z. Phys. C70 (1996) 65.

[34] G. Mills et al. (DELCO Collaboration), Phys. Rev. Lett. 54 (1985) 624.

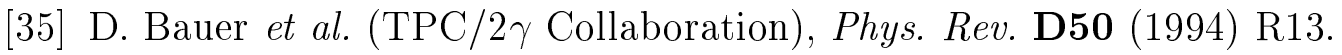

[36] A. Pais, Ann. Phys. 9 (1960) 548.

[37] V. Shelkov (for the CLEO Collaboration), 'Tau decays involving $\eta$ mesons and $\tau^{-} \rightarrow \pi^{-} \mathrm{f}_{1} \nu_{\tau}$, Talk given at the TAU'96 Conference, Colorado (1996).

[38] D. Buskulic et al. (ALEPH Collaboration), 'ALEPH Handbook Vol. 1', ed. by C. Bowdery, (1995)

[39] S. Eidelman and V.N. Ivanchenko, Phys. Lett. B257 (1991) 437.

[40] S. Eidelman and V.N. Ivanchenko, Nucl. Phys. B40 (Proc. Suppl.) (1995) 131.

[41] A. Dobado, M.J. Herrero, T.N. Truong, Phys. Lett. B235 (1990) 134.

[42] J. Gasser and U.G. Meissner, Nucl. Phys. B357 (1991) 90.

[43] S. Eidelman and F. Jegerlehner, Z. Phys. C67 (1995) 585.

[44] L.M. Barkov et al. (OLYA, CMD Collaboration), Nucl. Phys. B256 (1985) 365.

[45] I.B. Vasserman et al. (OLYA Collaboration), Sov. J. Nucl. Phys. 30 (1979) 519.

[46] I.B. Vasserman et al. (TOF Collaboration), Sov. J. Nucl. Phys. 33 (1981) 709.

[47] S.R. Amendolia et al. (NA7 Collaboration), Phys. Lett. B138 (1984) 454.

[48] A. Quenzer et al. (DM1 Collaboration), Phys. Lett. B76 (1987) 512.

[49] D. Bisello et al. (DM2 Collaboration), Phys. Lett. B220 (1989) 321.

[50] B. Esposito et al. (MEA Collaboration), Phys. Lett. B67 (1977) 239; Lett. Nuovo Cim. 28 (1980) 337.

[51] D. Bollini et al. (BCF Collaboration), Lett. Nuovo Cim. 14 (1975) 418.

[52] M. Bernardini et al. (BCF Collaboration), Phys. Lett. B46 (1973) 261.

[53] S.R. Amendolia et al. (NA7 Collaboration), Nucl. Phys. B277 (1986) 168.

[54] G. Colangelo, M. Finkemeier and R. Urech, 'Tau Decays and Chiral Perturbation Theory', BUTP-96/9 HUTP95/-A051, TTP96-02, hep-ph/9604279 (1996).

[55] L.M. Kurdadze et al. (OLYA Collaboration), JETP Lett. 43 (1986) 643.

[56] S.J. Dolinsky et al. (ND Collaboration), Phys. Rep. C202 (1991) 99.

[57] B. Esposito et al. (MEA Collaboration), Lett. Nuovo Cim. 28 (1980) 195. 
[58] L.M. Barkov et al. (CMD Collaboration), Sov. J. Nucl. Phys. 47 (1988) 248.

[59] A. Cordier et al. (DM1 Collaboration), Phys. Lett. B109 (1981) 155.

[60] A. Cordier et al. (DM1 Collaboration), Phys. Lett. B81 (1979) 389.

[61] D. Bisello (for the DM2 Collaboration), Nucl. Phys. B21 (Proc. Suppl.) (1995) 111.

[62] D. Bisello et al. (DM2 Collaboration), Report LAL-90-35, Orsay (1990).

[63] L. Stanco (for the DM2 Collaboration), Proceedings of Hadron-91 (World Scientific ed. 1992) 84.

[64] G. Cosme et al. (M3N Collaboration), Nucl. Phys. B152 (1979) 215; C. Paulot, Thesis, Report LAL-79-14, Orsay (1979).

[65] G. Cosme et al. (M2N Collaboration), Phys. Lett. B63 (1976) 349; G. Parrour et al. (M2N Collaboration), Phys. Lett. B63 (1976) 357.

[66] A. Antonelli et al. (DM2 Collaboration), Phys. Lett. B212 (1988) 133.

[67] D. Bisello et al. (DM1 Collaboration), Phys. Lett. B107 (1981) 145.

[68] M. Schioppa (DM2 Collaboration), Thesis, Universita di Roma "La Sapienza" (1986).

[69] F. Mane et al. (DM1 Collaboration), Phys. Lett. B112 (1982) 178.

[70] D. Bisello et al. (DM2 Collaboration), Z. Phys. C52 (1991) 227.

[71] F. Mané (DM1 Collaboration), Thesis, Université de Paris-Sud, Report LAL 82/46 (1982).

[72] A. Cordier et al. (DM1 Collaboration), Phys. Lett. B110 (1982) 335.

[73] M. Benayoun et al., Z. Phys. C58 (1993) 31.

[74] G. D’Agostini, Nucl. Inst. Meth. A346 (1994) 306.

[75] B. Costa de Beauregard, T.N. Pham and T.N. Truong, Phys. Lett. B67 (1977) 213.

[76] A.B. Clegg and A. Donnachie, Z. Phys. C62 (1994) 455.

[77] K. Huber and H. Neufeld, Phys. Lett. B357 (1995) 221.

[78] J. Bijnens and P. Gosdzinsky, Phys. Lett. B388 (1996) 203.

[79] J. Gasser and H. Leutwyler, Phys. Rep. 87 (1982) 77.

[80] L.P. Chen and W. Dunwoodie (for the MARK III Collaboration), Proceedings of Hadron-91 (World Scientific ed. 1992) 100.

[81] R.J. Sobie, Z. Phys. C69 (1995) 99. 